\title{
A Hybrid Evolutionary Algorithm for Heterogeneous Fleet Vehicle Routing Problems with Time Windows
}

\author{
Çağrı Koça ${ }^{\mathrm{a}}$ Tolga Bektaş̧a ${ }^{\mathrm{a}}$ Ola Jabalib,*, Gilbert Laporte ${ }^{\mathrm{c}}$ \\ ${ }^{a}$ CORMSIS and Southampton Business School, University of Southampton, Southampton SO17 1BJ, \\ United Kingdom \\ ${ }^{b}$ CIRRELT and HEC Montréal, 3000, chemin de la Côte-Sainte-Catherine, Montréal H3T 2A7, Canada \\ ${ }^{c}$ CIRRELT and Canada Research Chair in Distribution Management, HEC Montréal, 3000, chemin de la \\ Côte-Sainte-Catherine, Montréal H3T 2A7, Canada
}

\begin{abstract}
This paper presents a hybrid evolutionary algorithm (HEA) to solve heterogeneous fleet vehicle routing problems with time windows. There are two main types of such problems, namely the Fleet Size and Mix Vehicle Routing Problem with Time Windows (F) and the Heterogeneous Fixed Fleet Vehicle Routing Problem with Time Windows (H), where the latter, in contrast to the former, assumes a limited availability of vehicles. The main objective is to minimize the fixed vehicle cost and the distribution cost, where the latter can be defined with respect to en-route time $(\mathrm{T})$ or distance $(\mathrm{D})$. The proposed unified algorithm is able to solve the four variants of heterogeneous fleet routing problem, called FT, FD, HT and HD, where the last variant is new. The HEA successfully combines several metaheuristics and offers a number of new advanced efficient procedures tailored to handle the heterogeneous fleet dimension. Extensive computational experiments on benchmark instances have shown that the HEA is highly effective on FT, FD and HT. In particular, out of the 360 instances we obtained 75 new best solutions and matched 102 within reasonable computational times. New benchmark results on HD are also presented.
\end{abstract}

Keywords: vehicle routing, time windows, heterogeneous fleet, genetic algorithm, neighborhood search

\footnotetext{
*Corresponding author. Tel.: 514 340-6154

Email addresses: C.Koc@soton.ac.uk (Çağrı Koç), T.Bektas@soton.ac.uk (Tolga Bektaş), Ola.Jabali@hec.ca (Ola Jabali), Gilbert.Laporte@cirrelt.ca (Gilbert Laporte)
} 


\section{Introduction}

In heterogeneous fleet vehicle routing problems with time windows, one considers a fleet of vehicles with various capacities and vehicle-related costs, as well as a set of customers with known demands and time windows. These problems consist of determining a set of vehicle routes such that each customer is visited exactly once by a vehicle within a prespecified time window, all vehicles start and end their routes at a depot, and the load of each vehicle does not exceed its capacity. As is normally the case in vehicle routing problem with time windows (VRPTW), customer service must start within the time window, but the vehicle may wait at a customer location if it arrives before the beginning of the time window. There are two main categories of such problems, namely the Fleet Size and Mix Vehicle Routing Problem with Time Windows (F) and the Heterogeneous Fixed Fleet Vehicle Routing Problem with Time Windows $(\mathrm{H})$. In category $\mathrm{F}$, there is no limit in the number of available vehicles of each type, whereas such a limit exists in category H. Note that it is easy to find feasible solutions to the instances of category $\mathrm{F}$ since there always exists a feasible assignment of vehicles to routes. However, this is not always the case for the instances of category $\mathrm{H}$.

Two measures are used to compute the total cost to be minimized. The first is the sum of the fixed vehicle cost and of the en-route time $(\mathrm{T})$, which includes traveling time and possible waiting time at the customer locations before the opening of their time windows (we assume that travel time and cost are equivalent). In this case, service times are only used to check feasibility and for performing adjustments to the departure time from the depot in order to minimize pre-service waiting times. The second cost measure is based on distance (D) and consists of the fixed vehicle cost and the distance traveled by the vehicle, as is the case in the standard VRPTW (Solomon, 1987).

We differentiate between four variants defined with respect to the problem category and to the way in which the objective function is defined, namely FT, FD, HT and HD. The first variant is FT, described by Liu and Shen (1999b) and the second is FD, introduced by Bräysy et al. (2008). The third variant HT was defined and solved by Paraskevopoulos et al. (2008). Finally, HD is a new variant which we introduce in this paper. HD differs from HT 
by considering the objective function $\mathrm{D}$ instead of $\mathrm{T}$. This variant has never been studied before.

Hoff et al. (2010) and Belfiore and Yoshizaki (2009) describe several industrial aspects and practical applications of heterogeneous vehicle routing problems. The most studied versions are the fleet size and mix vehicle routing problem, described by Golden et al. (1984), which considers an unlimited heterogeneous fleet, and the heterogeneous fixed fleet vehicle routing problem, proposed by Taillard (1999). For further details, the reader is referred to the surveys of Baldacci et al. (2008) and of Baldacci and Mingozzi (2009).

The FT variant has several extensions, e.g., multiple depots (Dondo et al., 2007; Bettinelli et al., 2011), overloads (Kritikos and Ioannou, 2013), and split deliveries (Belfiore and Yoshizaki, 2009, 2013). There exist several exact algorithms for the capacitated vehicle routing problem (VRP) (Toth and Vigo, 2002; Baldacci et al., 2010), and for the heterogeneous VRP (Baldacci and Mingozzi, 2009). However, to the best of our knowledge, no exact algorithm has been proposed for the heterogeneous VRP with time windows, i.e., FT, FD and HT. The existing heuristic algorithms for these three variants are briefly described below.

Liu and Shen (1999b) proposed a heuristic for FT which starts by determining an initial solution through an adaptation of the Clarke and Wright (1964) savings algorithm, previously presented by Golden et al. (1984). The second stage improves the initial solution by moving customers by means of parallel insertions. The algorithm was tested on a set of 168 benchmark instances derived from the set of Solomon (1987) for the VRPTW. Dullaert et al. (2002) described a sequential construction algorithm for FT, which is an extension of the insertion heuristic of Golden et al. (1984). Dell'Amico et al. (2007) described a multi-start parallel regret construction heuristic for FT, which is embedded into a ruin and recreate metaheuristic. Bräysy et al. (2008) presented a deterministic annealing metaheuristic for FT and FD. In a later study, Bräysy et al. (2009) described a hybrid metaheuristic algorithm for large scale FD instances. Their algorithm combines the well-known threshold acceptance heuristic with a guided local search metaheuristic having several search limitation strategies. An adaptive memory programming algorithm was proposed by Repoussis and 
Tarantilis (2010) for FT, which combines a probabilistic semi-parallel construction heuristic, a reconstruction mechanism and a tabu search algorithm. Computational results indicate that their method is highly successful and improves many best known solutions. In a recent study, Vidal et al. (2014) introduced a genetic algorithm based on a unified solution framework for different variants of the VRPs, including FT and FD. To our knowledge, Paraskevopoulos et al. (2008) are the only authors who have studied HT. Their two-phase solution methodology is based on a hybridized tabu search algorithm capable of solving both FT and HT.

This brief review shows that the two problem categories $\mathrm{F}$ and $\mathrm{H}$ have already been solved independently through different methodologies. We believe there exists merit for the development of a unified algorithm capable of efficiently solving the two problem categories. This is the main motivation behind this paper.

This paper makes three main scientific contributions. First, we develop a unified hybrid evolutionary algorithm (HEA) capable of handling the four variants of the problem. The HEA combines two state-of-the-art metaheuristic concepts which have proved highly successful on a variety of VRPs: Adaptive Large Neighborhood Search (ALNS) (see Ropke and Pisinger, 2006a; Pisinger and Ropke, 2007; Demir et al., 2012) and population based search (see Prins, 2004; Vidal et al., 2014). The second contribution is the introduction of several algorithmic improvements to the procedures developed by Prins (2009) and Vidal et al. (2012). We use a ALNS equipped with a range of operators as the main EDUCATION procedure within the search. We also propose an advanced version of the Split algorithm of Prins (2009) capable of handling infeasibilities. Finally, we introduce an innovative aggressive INTENSIFICATION procedure on elite solutions, as well as a new diversification scheme through the Regeneration and the Mutation procedures of solutions. The third contribution is to introduce HD as a new problem variant.

The remainder of this paper is structured as follows. Section 2 presents a detailed description of the HEA. Computational experiments are presented in Section 3, and conclusions are provided in Section 4. 


\section{Description of the Hybrid Evolutionary Algorithm}

We start by introducing the notation related to FT, FD, HT and HD. All problems are defined on a complete graph $G=(N, A)$, where $N=\{0, \ldots, n\}$ is the set of nodes, and node 0 corresponds to the depot. Let $A=\{(i, j): 0 \leq i, j\} \leq n, i \neq j\}$ denote the set of arcs. The distance from $i$ to $j$ is denoted by $d_{i j}$. The customer set is $N_{c}$ in which each customer $i$ has a demand $q_{i}$ and a service time $s_{i}$, which must start within time window $\left[a_{i}, b_{i}\right]$. If a vehicle arrives at customer $i$ before $a_{i}$, it then waits until $a_{i}$. Let $K=\{1, \ldots, k\}$ be the set of available vehicle types. Let $e_{k}$ and $Q_{k}$ denote the fixed vehicle cost and the capacity of vehicle type $k$, respectively. The travel time from $i$ to $j$ is denoted by $t_{i j}$ and is independent of the vehicle type. The distribution cost from $i$ to $j$ associated with a vehicle of type $k$ is $c_{i j}^{k}$ for all problem types. In HT and HD, the available number of vehicles of type $k \in K$ is $n_{k}$, whereas the constant can be set to an arbitrary large value for problems FT and FD. The objectives are as discussed in the Introduction.

The remainder of this section introduces the main components of the HEA. A general overview of the HEA is given in Section 2.1. More specifically, Section 2.2 presents the offspring EDUCATION procedure. Section 2.3 presents the initialization of the population. The selection of parent solutions, the ordered crossover operator and the advanced algorithm SPLIT are described in Sections 2.4, 2.5 and 2.6, respectively. Section 2.7 presents the INTENSIFICATION procedure. The survivor selection mechanism is detailed in Section 2.8. Finally, the diversification stage, including the REGENERATION and MutATION procedures, is described in Section 2.9.

\subsection{Overview of the Hybrid Evolutionary Algorithm}

The general structure of the HEA is presented in Algorithm 1. The modified version of the classical Clarke and Wright savings algorithm and the ALNS operators are combined to generate the initial population (Line 1). Two parents are selected (Line 3) through a binary tournament, following which the crossover operation (Line 4) generates a new offspring $C$. The advanced SPLIT algorithm is applied to the offspring $C$ (Line 5), which optimally segments the giant tour by choosing the vehicle type for each route. The EDUCATION 


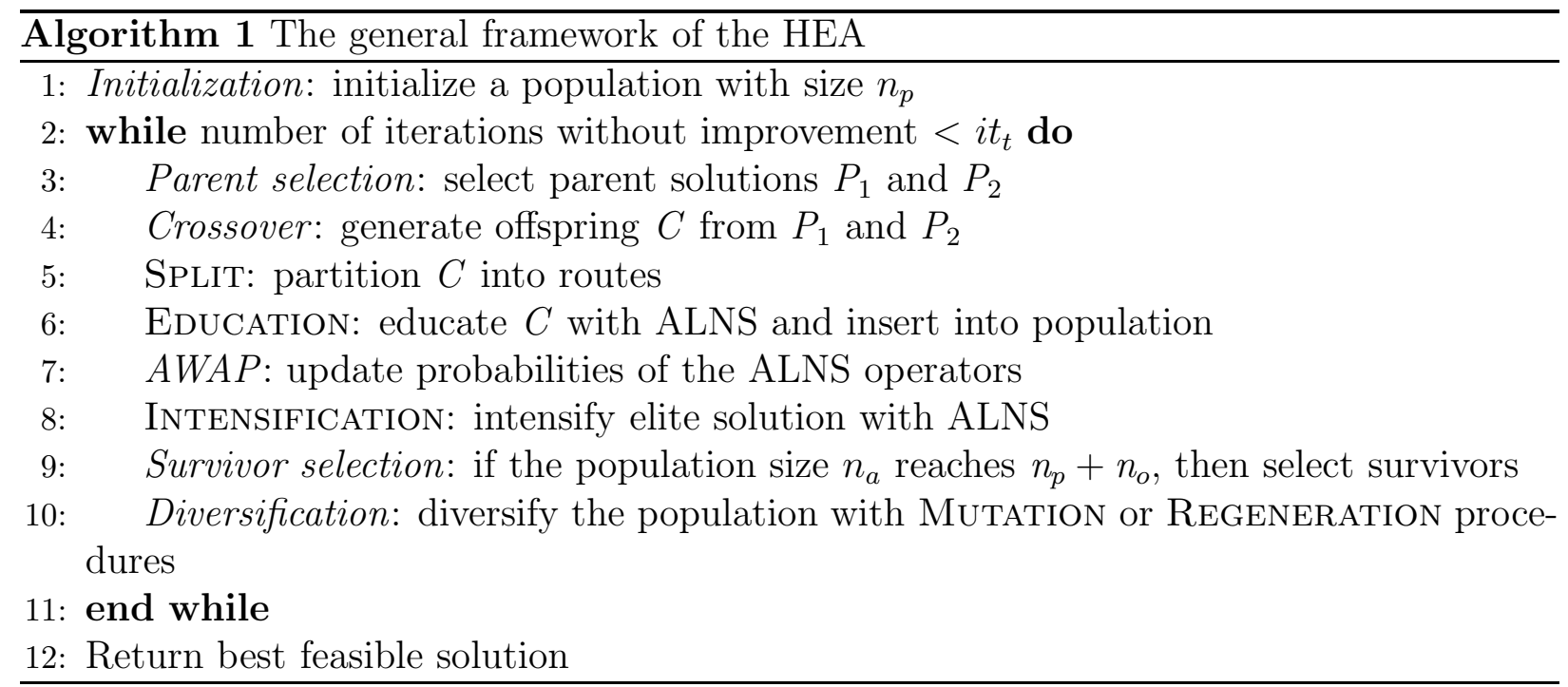




\subsection{EDUCATION}

The EDUCATION procedure is systematically applied to each offspring in order to improve its quality. The ALNS algorithm is used as a way of educating the solutions in the HEA. This is achieved by applying both the destroy and repair operators, and a number of removable nodes are modified in each iteration. An example of the removal and insertion phases is illustrated in Figure 1. The operators used within the HEA are either adapted or inspired from those employed by various authors (Ropke and Pisinger, 2006a,b; Pisinger and Ropke, 2007; Demir et al., 2012; Paraskevopoulos et al., 2008).

a: A feasible solution

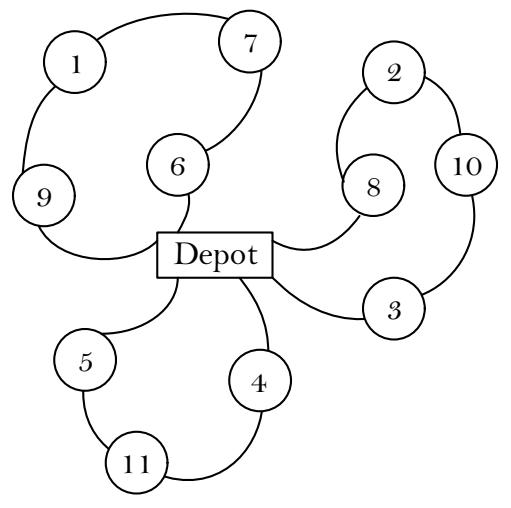

$L_{r}=\varnothing$ b: A destroyed solution

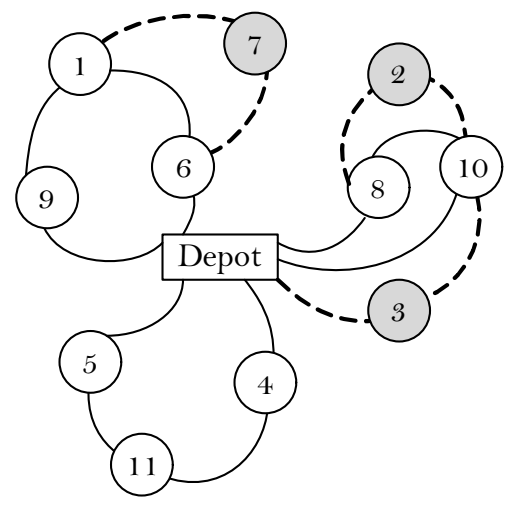

$L_{r}=\{7,2,3\}$ c: A repaired solution

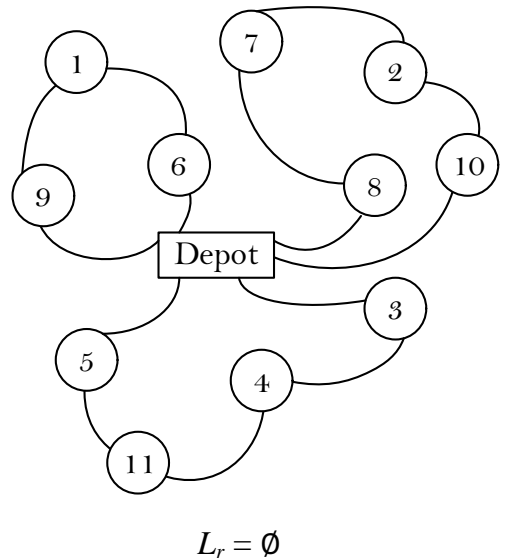

Figure 1: Illustration of the EdUCATION procedure

The Education procedure is detailed in Algorithm 2. All operators are repeated $O(n)$ times and the complexity given are the overall repeats. The removal procedure (line 4 of Algorithm 2) runs for $n^{\prime}$ iterations, removes $n^{\prime}$ customers from the solution and add to the removal list $L_{r}$, where $n^{\prime}$ is in the interval of removable nodes $\left[b_{l}^{e}, b_{u}^{e}\right]$. An insertion operator is then selected to iteratively insert the nodes, starting from the first customer of $L_{r}$, into the partially destroyed solution until $L_{r}$ is empty (line 5 ). The feasibility conditions in terms of capacity and time windows for FT, FD, HT and HD, and in terms of fleet size for HT and HD, are always respected during the insertion process. We do not allow overcapacity of the vehicle and service start outside the time windows for all problem types, and we also do not allow the use of additional vehicles beyond the fixed fleet size for HT and HD. The 
removal and insertion operators are randomly selected according to their past performance and a certain probability as explained further in Section 2.2.3. The cost of an individual $C$ before the removal is denoted by $\omega(C)$, and its cost after the insertion is denoted by $\omega\left(C^{*}\right)$.

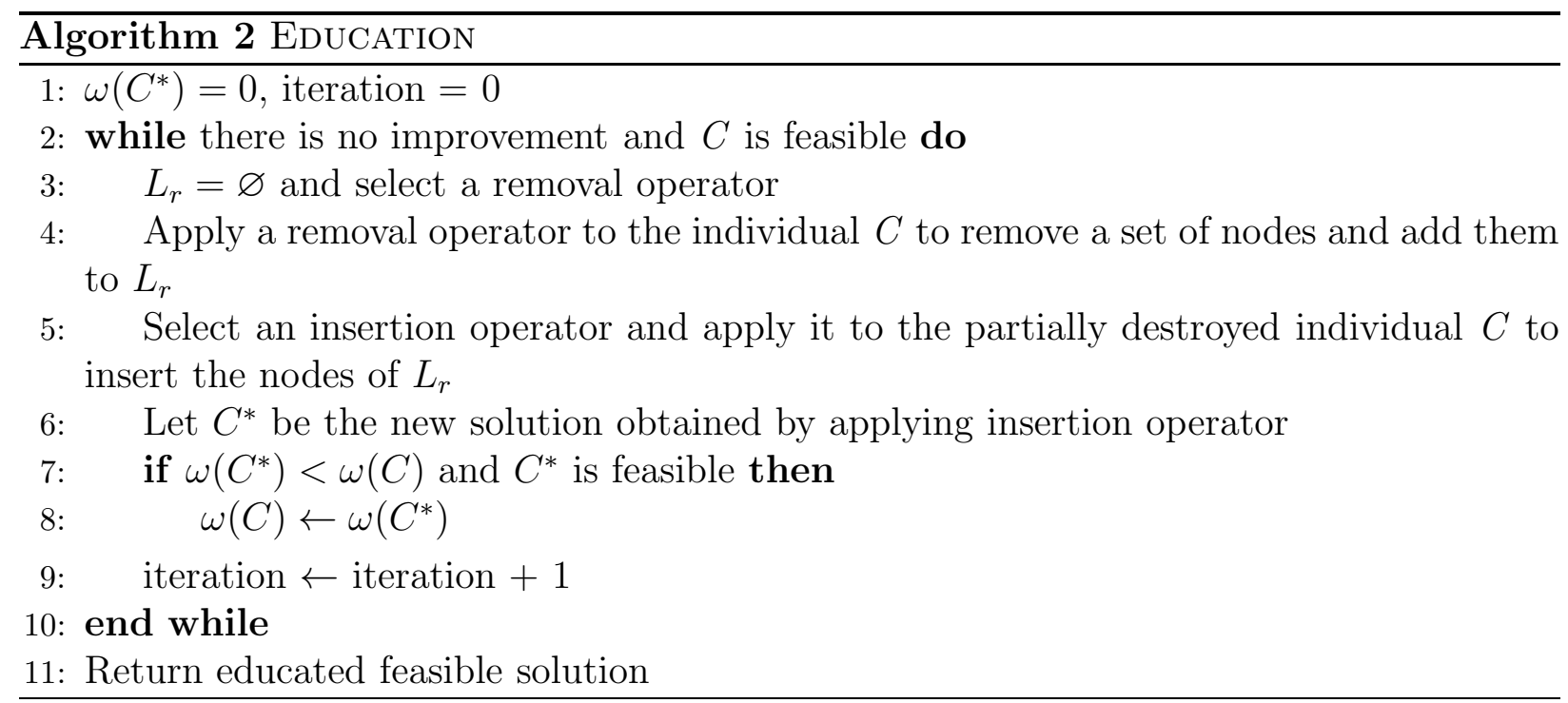

The heterogeneous fleet version of the ALNS that we use here was recently introduced by Koç et al. (2014). It educates solutions by considering the heterogeneous fleet aspect. The ALNS integrates fleet sizing within the destroy and repair operators. In particular, if a node is removed, we check whether the resulting route can be served by a smaller vehicle. We then update the solution accordingly. If inserting a node requires additional vehicle capacity we then consider the option of using larger vehicles. For each node $i \in N_{c} \backslash L_{r}$, let $f^{h}(i)$ be the current vehicle fixed cost associated with the vehicle serving $i$. Let $\Delta(i)$ be the saving obtained as a result of using a removal operator on node $i$ without considering the vehicle fixed cost. Let $f_{1}^{h *}(i)$ be the vehicle fixed cost after removal of node $i$. Consequently, $f_{1}^{h *}(i)<f^{h}(i)$ only if the route containing node $i$ can be served by a smaller vehicle when removing node $i$. The savings in vehicle fixed cost can be expressed as $f^{h}(i)-f_{1}^{h *}(i)$, respectively. Thus, for each removal operator, the total savings of removing node $i \in N_{c} \backslash L_{r}$, denoted $R C(i)$, is calculated as follows:

$$
R C(i)=\Delta(i)+\left(f^{h}(i)-f_{1}^{h *}(i)\right)
$$


In a destroyed solution, the insertion cost of node $j \in L_{r}$ after node $i$ is defined as $\Omega(i, j)$ for a given node $i \in N_{c} \backslash L_{r}$. Let $f_{2}^{h *}(i)$ be the vehicle fixed cost after the insertion of node $i$, i.e., $f_{a}^{h *}>f^{h}$ only if the route containing node $i$ necessitates the use of a larger capacity vehicle after inserting node $i$. The cost differences in vehicle fixed cost can be expressed as $f_{2}^{h *}(i)-f^{h}(i)$. Thus, the total insertion cost of node $i \in N_{c} \backslash L_{r}$, for each insertion operator is

$$
I C(i)=\Omega(i, j)+\left(f_{2}^{h *}(i)-f^{h}(i)\right) .
$$

\subsubsection{Removal Operators}

Nine removal operators are used in the destroy phase of the EDUCATION procedure and are described in detail below.

1.Random removal (RR): The RR operator randomly selects a node $j \in N \backslash\{0\} \backslash L_{r}$, removes it from the solution. The worst-case time complexity of the RR operator is $O(n)$.

2. Worst distance removal (WDR): The purpose of the WDR operator is to choose a number of expensive nodes according to their distance based cost. The cost of a node $j \in$ $N \backslash\{0\} \backslash L_{r}$ is the distance from its predecessor $i$ and its distance to its successor $k$. The WDR operator iteratively removes nodes $j^{*}$ from the solution where $j^{*}=\arg \max _{j \in N \backslash\{0\} \backslash L_{r}}\left\{d_{i j}+\right.$ $\left.d_{j k}+f^{h}(i)-f_{1}^{h *}(i)\right\}$. The time complexity of this operator is $O\left(n^{2}\right)$.

3. Worst time removal (WTR): The WTR operator is a variant of the WDR operator. For each node $j \in N \backslash\{0\} \backslash L_{r}$ costs are calculated, depending on the deviation between the arrival time $z_{j}$ and the beginning of the time window $a_{j}$. The WTR operator iteratively removes customers from the solution, where $j^{*}=\arg \max _{j \in N \backslash\{0\} \backslash L_{r}}\left\{\left|z_{j}-a_{j}\right|+f^{h}(i)-f_{1}^{h *}(i)\right\}$. The ALNS iteratively applies this process to the solution after each removal. The WTR operator can be implemented in $O\left(n^{2}\right)$ time.

4. Neighborhood removal (NR): In a given solution with a set $\Re$ of routes, the NR operator calculates an average distance $\bar{d}(R)=\sum_{(i, j) \in R} d_{i j} /|R|$ for each route $R \in \Re$, and selects a node $j^{*}=\arg \max _{(R \in \Re ; j \in R)}\left\{\bar{d}(R)-d_{R \backslash\{j\}}+f^{h}(i)-f_{1}^{h *}(i)\right\}$, where $d_{R \backslash\{j\}}$ denotes the average distance of route $R$ excluding node $j$. The time complexity of this operator is $O\left(n^{2}\right)$.

5. Shaw removal (SR): The general idea behind the SR operator, which was introduced 
by Shaw (1998), is to remove a set of customers that are related in a predefined way and are therefore easy to change. The SR operator removes a set of $n^{\prime}$ similar customers. The similarity between two customers $i$ and $j$ is defined by the relatedness measure $\delta(i, j)$. This includes four terms: a distance term $d_{i j}$, a time term $\left|a_{i}-a_{j}\right|$, a relation term $l_{i j}$, which is equal to -1 if $i$ and $j$ are in the same route, and 1 otherwise, and a demand term $\left|q_{i}-q_{j}\right|$. The relatedness measure is given by

$$
\delta(i, j)=\varphi_{1} d_{i j}+\varphi_{2}\left|a_{i}-a_{j}\right|+\varphi_{3} l_{i j}+\varphi_{4}\left|q_{i}-q_{j}\right|,
$$

where $\varphi_{1}$ to $\varphi_{4}$ are weights that are normalized to find the best candidate solution. The operator starts by randomly selecting a node $i \in N \backslash\{0\} \backslash L_{r}$, and selects the node $j^{*}$ to remove where $j^{*}=\arg \min _{j \in N \backslash\{0\} \backslash L_{r}}\left\{\delta(i, j)+f^{h}(i)-f_{1}^{h *}(i)\right\}$. The operator is iteratively applied to select a node which is most similar to the one last added to $L_{r}$. The time complexity of this operator is $O\left(n^{2}\right)$.

6. Proximity-based removal (PBR): This operator is a second variant of the classical Shaw removal operator. The selection criterion of a set of routes is solely based on the distance. Therefore, the weights are $\varphi_{1}=1$ and $\varphi_{2}=\varphi_{3}=\varphi_{4}=0$. The PBR operator can be implemented in $O\left(n^{2}\right)$ time.

7. Time-based removal (TBR): The TBR operator removes a set of nodes that are related in terms of time. It is a special case of the Shaw removal operator where $\varphi_{2}=1$ and $\varphi_{1}=\varphi_{3}=\varphi_{4}=0$. Its time complexity is $O\left(n^{2}\right)$.

8. Demand-based removal (DBR): The DBR operator is yet another variant of the Shaw removal operator with $\varphi_{4}=1$ and $\varphi_{1}=\varphi_{2}=\varphi_{3}=0$. It can be implemented in $O\left(n^{2}\right)$ time.

9. Average cost per unit removal (ACUTR): The average cost per unit (ACUT) is described by Paraskevopoulos et al. (2008) to measure the utilization efficiency of a vehicle $\Pi(R)$ on a given route $R . \Pi(R)$ is expressed as the ratio of the total travel cost and fixed vehicle cost over the total demand carried by a vehicle $k$ traversing route $R$ :

$$
\Pi(R)=\frac{\sum_{(i, j) \in A} c_{i j} x_{i j}^{k}+e^{k}}{\sum_{i \in N \backslash\{0\}} q_{i} x_{i j}^{k}} .
$$


The aim of the ACUTR operator is to calculate the cost of each route and remove the one with the least $\Pi(R)$ value from the solution. The ACUTR operator can be implemented in $O\left(n^{2}\right)$ time.

\subsubsection{Insertion Operators}

Three insertion operators are used in the repair phase of the EDUCATION procedure.

1. Greedy insertion (GI): The aim of this operator is to find the best possible insertion position for all nodes in $L_{r}$. For node $i \in N \backslash L_{r}$ succeeded in the destroyed solution by $k \in N \backslash\{0\} \backslash L_{r}$, and node $j \in L_{r}$ we define $\gamma(i, j)=d_{i j}+d_{j k}-d_{i k}$. We find the least-cost insertion position for $j \in L_{r}$ by $i^{*}=\arg \min _{i \in N \backslash L_{r}}\left\{\gamma(i, j)+f_{2}^{h *}(i)-f^{h}(i)\right\}$. This process is iteratively applied to all nodes in $L_{r}$. The time complexity of this operator is $O\left(n^{2}\right)$.

2. Greedy insertion with noise function (GINF): The GINF operator is based on the GI operator but extends it by allowing a degree of freedom in selecting the best place for a node. This is done by calculating the noise cost $v(i, j)=\gamma(i, j)+f_{2}^{h *}(i)-f^{h}(i)+d_{\max } p_{n} \epsilon$ where $d_{\max }$ is the maximum distance between all nodes, $p_{n}$ is a noise parameter used for diversification and is set equal to 0.1 , and $\epsilon$ is a random number in $[-1,1]$. The time complexity of this operator is $O\left(n^{2}\right)$.

3. Greedy insertion with en-route time (GIET): This operator calculates the en-route time difference $\eta(i, j)$ between before and after inserting the customer $j \in L_{r}$. For node $i \in N \backslash L_{r}$ succeeded in the destroyed solution by $k \in N \backslash\{0\} \backslash L_{r}$, and node $j \in L_{r}$, we define $\eta(i, j)=\tau_{i j}+\tau_{j k}-\tau_{i k}$ where $\tau_{i j}$ is the en-route time from node $i$ to node $j$. We find the least-cost insertion position for $j \in L_{r}$ by $i^{*}=\arg \min _{i \in N \backslash L_{r}}\left\{\eta(i, j)+f_{2}^{h *}(i)-f^{h}(i)\right\}$. The GIET operator can be implemented in $O\left(n^{2}\right)$ time.

\subsubsection{Adaptive Weight Adjustment Procedure}

Each removal and insertion operator has a certain probability of being chosen in every iteration. The selection criterion is based on the historical performance of every operator and is calibrated by a roulette-wheel mechanism (Ropke and Pisinger, 2006a; Demir et al., 2012). After $i t_{w}$ iterations of the roulette wheel segmentation, the probability of each operator is recalculated according to its total score. Initially, the probabilities of each removal and 
insertion operator are equal. Let $p_{i}^{t}$ be the probability of operator $i$ in the last $i t_{w}$ iterations, $p_{i}^{t+1}=p_{i}^{t}\left(1-r_{p}\right)+r_{p} \pi_{i} / \tau_{i}$, where $r_{p}$ is the roulette wheel probability, for operator $i$; $\pi_{i}$ is its score and $\tau_{i}$ is the number times it was used during the last segment. At the start of each segment, the scores of all operators are set to zero. The scores are changed by $\sigma_{1}$ if a new best solution is found, by $\sigma_{2}$ if the new solution is better than the current solution and by $\sigma_{3}$ if the new solution is worse than the current solution.

\subsection{Initialization}

The procedure used to generate the initial population is based on a modified version of the Clarke and Wright and ALNS algorithms. An initial individual solution is obtained by applying Clarke and Wright algorithm and by selecting the largest vehicle type for each route. Then, until the initial population size reaches $n_{p}$, new individuals are created by applying to the initial solution operators based on random removals and greedy insertions with a noise function (see Section 2.2). We have selected these two operators in order to diversify the initial population. The number of nodes removed is randomly chosen from the initialization interval $\left[b_{l}^{i}, b_{u}^{i}\right]$, which is defined by a lower and an upper bound calculated as a percentage of the total number of nodes in an instance.

\subsection{Parent Selection}

In evolutionary algorithms, the evaluation function of individuals is often based on the solution cost. However, this kind of evaluation, does not take into account other important factors such as the diversity of the population which plays a critical role. Vidal et al. (2012) proposed a new method, named biased fitness $b f(C)$, to tackle this issue. This method considers the cost of an individual $C$, as well as its diversity contribution $d c(C)$ to the population. This function is continuously updated and is used to measure the quality of individuals during selection phases. The $d c(C)$ is defined as

$$
d c(C)=\frac{1}{n_{c}} \sum_{C^{\prime} \in N_{c}} \beta\left(C, C^{\prime}\right)
$$


where $N_{c}$ is the set of the $n_{c}$ closest neighbours of $C$ in the population. Thus, $d c(C)$ calculates the average distance between $C$ and its neighbours in $N_{c}$. The distance between two parents $\beta\left(C, C^{\prime}\right)$ is the number of pairs of adjacent requests in $C$ which are no longer adjacent, (called broken), in $C^{\prime}$. For example, let $C=\{4,5,6,7,8,9,10\}$ and $C^{\prime}=\{10,7,8,9,5,6,4\}$, in $C^{\prime}$ the pairs $\{4,5\},\{6,7\}$ and $\{9,10\}$ are broken and $\beta\left(C, C^{\prime}\right)=3$. The algorithm selects the broken pairs distance (see Prins, 2009) to compute the distance $\beta$. The main idea behind $d c(C)$ is to assess the differences between individuals.

The evaluation function of an individual $C$ in a population is

$$
b f(C)=r_{c}(C)+\left(1-\frac{n_{e}}{n_{a}}\right) r_{d c}(C),
$$

which is based on the rank $r_{c}(C)$ of solution cost, and on the rank $r_{d c}(C)$ of the diversity contribution. The rank $r_{d c}(C)$ is based on the diversity contribution calculated in equation (5), according to which the solutions are ranked in decreasing order of their $d c(C)$ value. In (6), $n_{e}$ is the number of elite individuals and $n_{a}$ is the current number of individuals.

The HEA selects two parents through a binary tournament to yield an offspring. The selection process randomly chooses two individuals from the population and keeps the one having the best biased fitness.

\subsection{Crossover}

Following the parent selection phase, two parents undergo the classical OrderEd Crossover or OX without trip delimiters. The OX operator is well suited for cyclic permutations, and the giant tour encoding allows recycling crossovers designed for the traveling salesman problem (TSP) (see Prins, 2004, 2009). Initially, two positions $i$ and $j$ are randomly selected in the first parent $P_{1}$. Subsequently, the substring $(i, \ldots, j)$ is copied into the first offspring $O_{1}$, at the same positions. The second parent $P_{2}$ is then swept cyclically from position $j+1$ onwards to fill the empty positions in $O_{1}$. The second offspring $O_{2}$ is generated likewise by exchanging the roles of $P_{1}$ and $P_{2}$. In the original version of $\mathrm{OX}$, two offsprings are obtained. However in the HEA, we only randomly select one offspring. 


\subsection{SPLIT Algorithm}

This algorithm is a tour splitting procedure which optimally partitions a solution into feasible routes. Each solution is a permutation of customers without trip delimiters and can therefore be viewed as a giant TSP tour for a vehicle with a large enough capacity. This algorithm was successfully applied in evolutionary based algorithms for several routing problems (Prins, 2004, 2009; Vidal et al., 2012, 2013).

We propose an advanced tour splitting procedure, denoted by SPLIT, which is embedded in the HEA to segment a giant tour and to determine the fleet mix composition. This is achieved through a controlled exploration of infeasible solutions (see Cordeau et al., 2001 and Nagata et al., 2010), by relaxing the limits on time windows and vehicle capacities. Violations of these limits are penalized through an objective function containing extra terms to account for infeasibilities. This is in contrast to Prins (2009) who does not allow infeasibilities, and in turn solves a resource-constrained shortest path problem using dynamic programming to determine the best fleet mix on a given solution. Our implementation also differs from those of Vidal et al. (2013) since it allows for infeasibilities that are not just related to time windows or load, but also to the fleet size in the case of HT and HD.

We now describe the SpLIT algorithm. Let $\Re$ be the set of all routes in individual $C$, and let $R$ be a route. While formally $R$ is a vector, for convenience we denote the number of its components by $|R|$. Therefore, $R=\left(i_{0}=0, i_{1}, i_{2}, \ldots, i_{|R|-1}, i_{|R|}=0\right)$, we also write $i \in R$ if $i$ is a component of $R$, and $(i, j) \in R$ if $i$ and $j$ appear in succession in $R$. Let $z_{t}$ be the arrival time at the $t^{\text {th }}$ customer in $R$, thus the time window violation of route $R$ is $\sum_{t=1}^{|R|-1} \max \left\{z_{t}-b_{i_{t}}, 0\right\}$. The total load for route $R$ is $\sum_{t=1}^{|R|-1} q_{i_{t}}$, and we consider solutions with a total load not exceeding twice the capacity of the largest vehicle given by $Q_{\max }$ (Vidal et al., 2013). Furthermore, for route $R$ and for each vehicle type $k$ we compute $y(k)$, which is the number of vehicles of type $k$ used in the solution.

Let $\lambda_{t}, \lambda_{l}$ and $\lambda_{f}$ represent the penalty values for any violations of the time windows, the vehicle capacity and the fleet size, respectively. The variable $x_{i j}^{k}$ is equal to 1 if customer $i$ immediately precedes customer $j$ visited by vehicle $k$. The fixed cost associated with using a vehicle of type $k \in K$ is denoted by $e_{k}$. For each route $R \in \Re$ traversed by vehicle $k \in K$, 
the cost including penalties is

$$
\nu(R, k)=\sum_{(i, j) \in R} c_{i j}^{k} x_{i j}^{k}+e_{k}+\lambda_{t} \sum_{t=1}^{|R|-1} \max \left\{z_{t}-b_{i_{t}}, 0\right\}+\lambda_{l} \max \left\{\sum_{t=1}^{|R|-1} q_{i_{t}}-Q_{\max }, 0\right\}
$$

which brings various objectives together to be able to guide to the search towards infeasible solutions. Thus, the total cost of individual $C$ is

$$
\Delta(C)=\sum_{R \in \Re} \sum_{k \in K} \nu(R, k)+\lambda_{f} \sum_{k \in K} \max \left\{0, y(k)-n_{k}\right\}
$$

where $n_{k}$ is set equal to a sufficiently large number (e.g., $n$ ) for FT and FD, in order for the last term in Equation (8) to be zero.

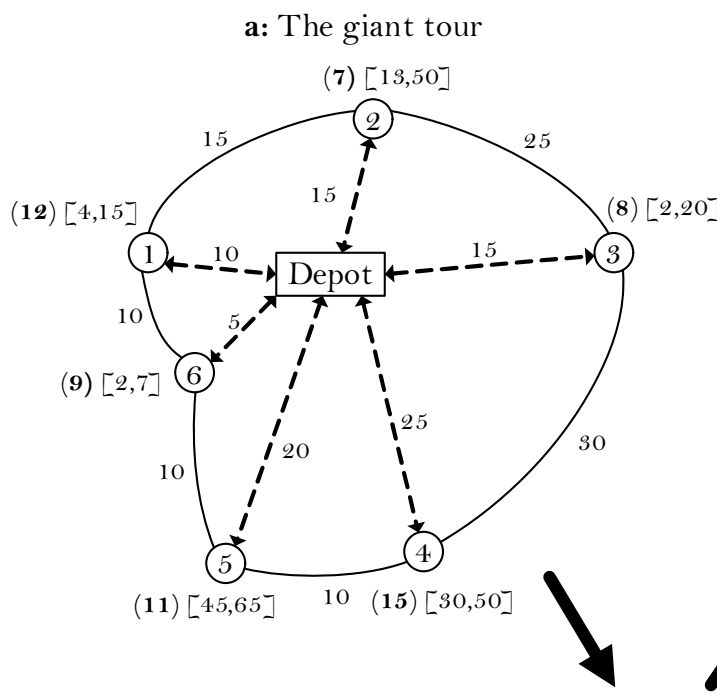

c: The optimal partition into routes and vehicles

b: The arcs of the shortest path solution and Graph $H$

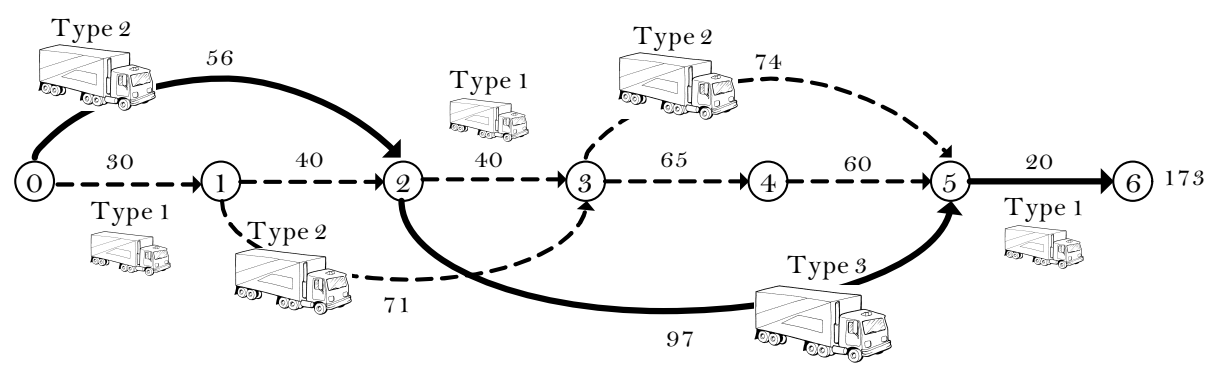

Figure 2: Illustration of procedure SPLIT

Figure 2 shows the steps of this advanced procedure using on an FD instance. The arc 
costs, demands and time windows are given in Figure 2.a. In particular, the number in bold within the parentheses associated with each node is the demand for that customer; the two numbers within brackets define the time window. Service times are identical and equal to 4 for each customer, and three different types of vehicles are available. The capacity $q_{k}$ and fixed cost $e_{k}$ of vehicles of type $\{1,2,3\}$ are $q_{1}=10, q_{2}=20, q_{3}=30$ and $e_{1}=6$, $e_{2}=8, e_{3}=10$, respectively. The algorithm starts with a giant TSP tour which includes six customers and uses one vehicle with unlimited capacity. The SPLIT algorithm computes an optimal compound segmentation in three routes corresponding to three sequences of customers $\{1,2\},\{3,4,5\}$ and $\{6\}$ with three vehicle choices, Type 2, Type 3 and Type 1, respectively, as shown in Figure 2.b. The resulting solution is shown in Figure 2.c. An optimal partitioning of the giant tour into routes for offspring $C$ corresponds to a minimumcost path.

The penalty parameters of the SPLIT algorithm are initially set to an initial value and are dynamically adjusted during the algorithm. If an individual is still infeasible after the first EDUCATION procedure, then the penalty parameters are multiplied by $\lambda_{m}$ and the EDUCATION procedure restarts. When this solution becomes feasible, the parameters are reset to their initial values. These values are $\lambda_{t}=\lambda_{l}=\lambda_{f}=3, \lambda_{m}=10$.

\subsection{INTENSIFICATION}

We introduce a two-phase aggressive INTENSIFICATION procedure to improve the quality of elite individuals. This procedure intensifies the search within promising regions of the solution space. The detailed pseude-code of this method is shown in Algorithm 3. The algorithm starts with an elite list of solutions $L_{e}$, which takes the best $n_{e}$ individuals from the main population as measured by equation (2). Step 1 is similar to the main EDUCATION procedure (Section 2.2). Step 2 attempts to explore different regions of the search space with the RR operator, intensifies this area by applying the GI operator for FD and HD, and GIET for FT and HT, to a partially the destroyed solution. Steps 1 and 2 terminate when there is no improvement to the solution and the main loop terminates when $n_{e}$ successive iterations have been performed. 
Due to the difficulty of the problems considered in this paper, we have developed a two-phase aggressive INTENSIFICATION procedure after having tried several variants such as one-phase with only Step 1 or Step 2, three-phase with Step 1, Step 2 and Step 1 and various other combinations. We have also considered other operators. Our analysis has shown that this two-phase structure yields better solutions than all other considered variants.

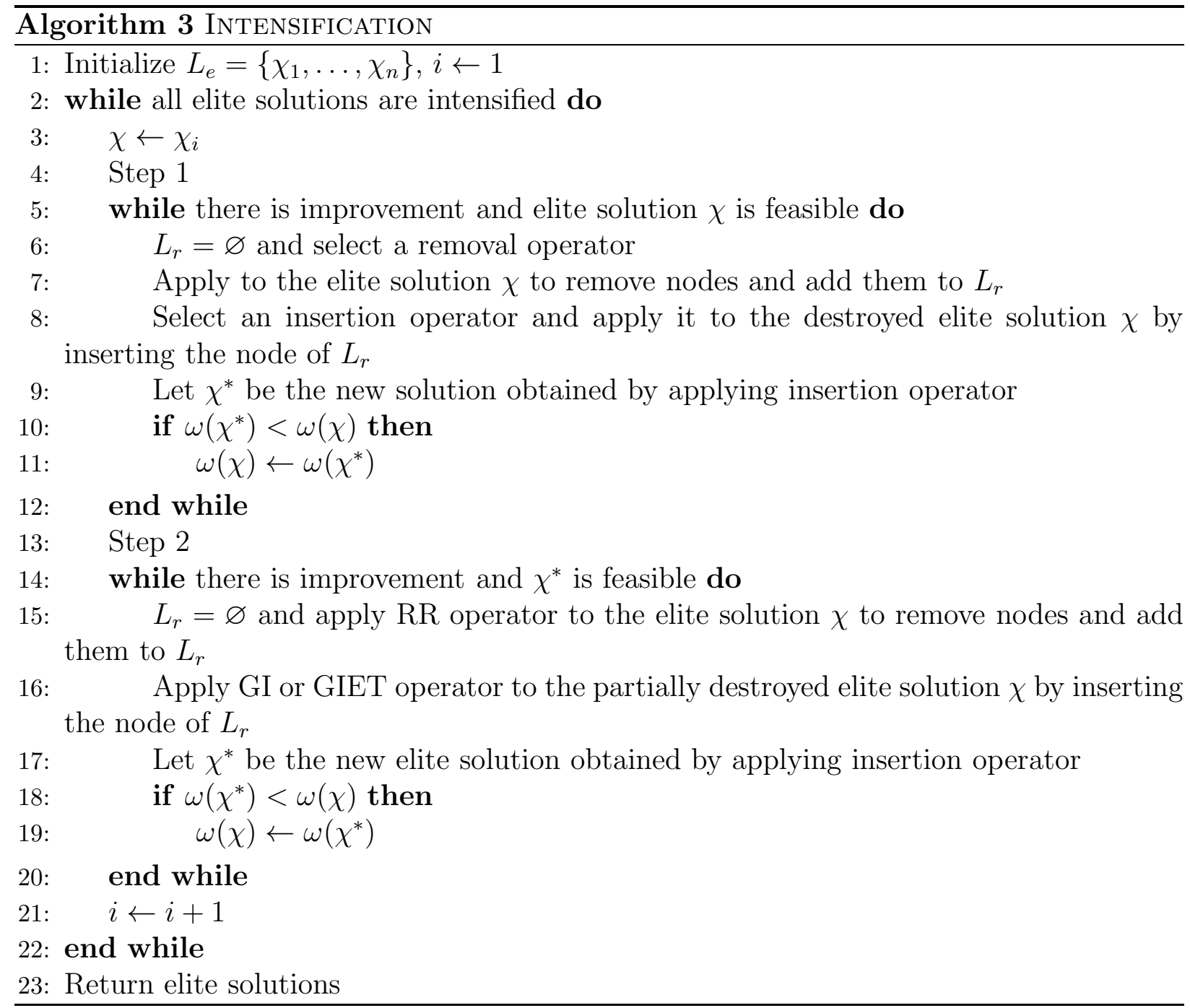

\subsection{Survivor Selection}

In population-based metaheuristics, avoiding premature convergence is a key challenge. Ensuring the diversity of the population, in other words to search a different location in the solution space during the algorithm, in the hope of being closer to the best known or optimal 
solutions constitutes a major trade-off between solutions in a population. The method of Vidal et al. (2012), aims to ensure the diversity of the population and preserve the elite solutions. The second part of this method is the survivor selection process (the first part was discussed in Section 2.3). In this way, elite individuals are protected.

\subsection{Diversification}

The efficient management of feasible solutions plays a significant role in population diversity. The performance of the HEA is improved by applying a MUTATION after the EDUCATION procedure. Over the iterations, individuals tend to become more similar, making it difficult to avoid premature convergence. To overcome this difficulty, we introduce a new scheme in order to increase the population diversity. The diversification stage includes two procedures, namely REGENERATION and MUTATION, representations of which are shown in Figure 3 .

a: REGENERATION
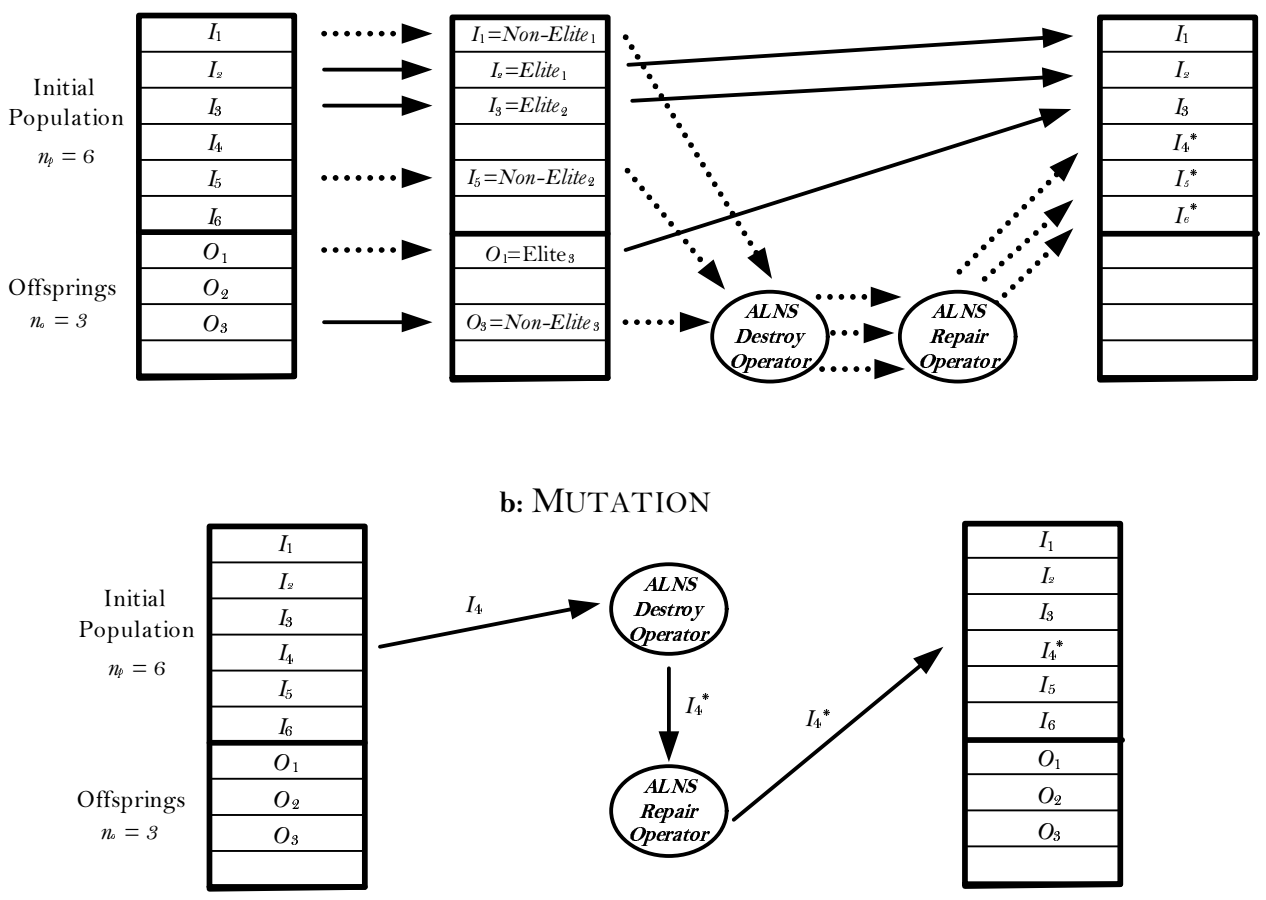

Figure 3: Illustration of the diversification stage

A RegenERAtion procedure (Figure 3.a) takes place when the maximum allowable 
iterations for REGENERATION $i t_{r}$ is reached without an improvement in the best solution value. In this procedure, the $n_{e}$ elite individuals are preserved and are transferred to the next generation. The remaining $n_{p}-n_{e}$ individuals, which are ranked according to their biased fitness, are subjected to the RR and GINF operators, to create new individuals. At the end of this procedure, only $n_{p}$ new individuals are kept in the population.

The Mutation procedure is applied with probability $p_{m}$. Figure 3.b illustrates the Mutation procedure. In this procedure, an individual $C$ different from the best solution is randomly selected. Two randomized structure based ALNS operators, the RR and the GINF, are then used to change the positions of a specific number of nodes, which are chosen from the interval $\left[b_{l}^{m}, b_{u}^{m}\right]$ of removable nodes in the Mutation procedure.

\section{Computational Experiments}

This section presents the results of computational experiments performed in order to assess the performance of the HEA. The HEA was implemented in $\mathrm{C}++$ and run on a computer with one gigabyte RAM and Intel Xeon $2.6 \mathrm{GHz}$ processor. We first describe the benchmark instances and the parameters used within the algorithm. This is followed by a presentation of the results.

\subsection{Data Sets and Experimental Settings}

The benchmark data sets of Liu and Shen (1999b), derived from the classical Solomon (1987) VRPTW instances with 100 nodes, are used as the test-bed. These sets include 56 instances, split into a random data set $\mathrm{R}$, a clustered data set $\mathrm{C}$ and a semi-clustered data set RC. Sets shown by R1, C1 and RC1 have a short scheduling horizon and small vehicle capacities, in contrast to sets denoted R2, C2 and RC2 with a long scheduling horizon and large vehicle capacities. Liu and Shen (1999b) introduced three types of cost structures, namely large, medium and small, and have denoted them by A, B and C, respectively. The authors also introduced several vehicle types with different capacities and fixed vehicle costs for each of the 56 instances. This results in a total of 168 benchmark instances for FT or FD. 
The benchmark set used by Paraskevopoulos et al. (2008) for HT is a subset of the FT instances, in which the fleet size is set equal to that found in the best known solutions of Liu and Shen (1999a). In total, there are 24 benchmark instances derived from Liu and Shen (1999a) for HT. We use the same set for HD, with the new objective.

Evolutionary algorithms use a set of correlated parameters and configuration decisions. In our implementation, we initially used the parameters suggested by Vidal et al. (2012, 2013) for the genetic algorithm, but we have conducted several experiments to further finetune these parameters on instances C101A, C203A, R101A, R211A, RC105A and RC207A. Following these tests, the following parameter values were used in our experiments: $i t_{t}=$ $5000, i t_{r}=2000, i t_{w}=500, n_{p}=25, n_{o}=25, n_{e}=10, n_{c}=3, p_{m} \in[0.4,0.6],\left[b_{l}^{i}, b_{u}^{i}\right]=$ $[0.3,0.8],\left[b_{l}^{e}, b_{u}^{e}\right]=[0.1,0.16],\left[b_{l}^{m}, b_{u}^{m}\right]=[0.1,0.16], \sigma_{1}=3, \sigma_{2}=1, \sigma_{3}=0$. For the Adaptive Large Neighborhood Search (ALNS), we have used the same parameter values as in Demir et al. (2012), namely $r_{p}=0.1, \varphi_{1}=0.5, \varphi_{2}=0.25, \varphi_{3}=0.15, \varphi_{4}=0.25$. All of these settings are identical for all four considered problems.

Table 1 presents the results of a fine-tuning experiment on parameters $n_{p}$ and $n_{o}$, and to test the effect of these parameters on the solution quality.

Table 1: Average percentage deviations of the solution values found by the HEA from best-known solution values with varying $n_{p}$ and $n_{o}$

\begin{tabular}{|l|lllll|}
\hline \multirow{2}{*}{$n_{p}$} & \multicolumn{5}{|c|}{$n_{o}$} \\
\cline { 2 - 6 } & 10 & 25 & 50 & 75 & 100 \\
\hline 10 & 0.42 & 0.26 & 0.38 & 0.56 & 0.69 \\
25 & 0.19 & $\mathbf{0 . 1 1}$ & 0.26 & 0.37 & 0.49 \\
50 & 0.39 & 0.29 & 0.30 & 0.45 & 0.57 \\
75 & 0.56 & 0.42 & 0.51 & 0.61 & 0.68 \\
100 & 0.67 & 0.53 & 0.61 & 0.72 & 0.78 \\
\hline
\end{tabular}

The table shows the percent gap between the solution value obtained by the HEA and the best-known solution (BKS) value, averaged over the six chosen instances. The maximum population size is dependent on $n_{p}$ and $n_{o}$, both of which have a significant impact on the solution quality, where the best setting is obtained with $n_{p}=n_{o}=25$. 


\subsection{Comparative Analysis}

We now present a comparative analysis of the results of the HEA with those reported in the literature. In particular, we compare ourselves against LSa (Liu and Shen, 1999a), LSb (Liu and Shen, 1999b), T-RR-TW (Dell'Amico et al., 2007), ReVNTS (Paraskevopoulos et al., 2008), MDA (Bräysy et al., 2008), BPDRT (Bräysy et al., 2009), AMP (Repoussis and Tarantilis, 2010) and UHGS (Vidal et al., 2014). The comparisons are presented in tables, where the columns show the total cost (TC), and percent deviations (Dev) of the values of solutions found by each method with respect to the HEA. The first column displays the instance sets and the number of instances in each set in parentheses. The rows named Avg (\%), Min (\%) and Max (\%) show the average, minimum and maximum deviations across all benchmark instances, respectively. A negative deviation shows that the solution found by the HEA is of better quality. In the column labeled BKS, "=" shows the total number of matches and " $<$ " shows the number of new BKS found for each instance set.

Ten separate runs are performed for each instance, the best one of which is reported. For each instance, a boldface refers to match with current BKS, where as a boldface with a "*" indicates new BKS. For detailed results, the reader is referred to Appendix A. Tables A.1-A.6 present the fixed vehicle cost (VC), the distribution cost (transportation cost) (DC), the computational time in minutes (Time) and the actual number of vehicles used (Mix), where the letters $\mathrm{A}-\mathrm{E}$ correspond to the vehicle types and the upper numbers denote the number of each type of vehicle used. For example, $\left(A^{2} B^{1}\right)$ indicates that two vehicles of type A and one vehicle of type B are used in the solution.

Tables 2 and 3 summarize the average comparison results of the current state-of-the-art solution methods for FT and FD, compared with the HEA. According to Tables 2 and 3, the HEA is highly competitive, with average deviations ranging from $-6.78 \%$ to $0.03 \%$ and a worst-case performance of $0.66 \%$ for FT. The average performance of our HEA is better than that of all the competitors for FT, except for the algorithm of Vidal et al. (2014) which is slightly better on average. However, the HEA found 17 new best solution and outperforms this algorithm on to the second type of FT instances, which are less tight in terms of vehicle capacity. As for FD, average cost reductions range from $-0.90 \%$ to $-0.02 \%$ and the worst- 
Table 2: Average results for FT

\begin{tabular}{|c|c|c|c|c|c|c|c|c|c|c|c|c|c|}
\hline \multirow[t]{2}{*}{ Instance set } & \multicolumn{2}{|c|}{ T-RR-TW } & \multicolumn{2}{|c|}{ ReVNTS } & \multicolumn{2}{|l|}{ MDA } & \multicolumn{2}{|l|}{ AMP } & \multicolumn{2}{|l|}{ UHGS } & \multirow{2}{*}{$\begin{array}{l}\text { HEA } \\
\text { TC }\end{array}$} & \multicolumn{2}{|c|}{ BKS } \\
\hline & TC & Dev & TC & Dev & TC & $\overline{\mathrm{Dev}}$ & TC & $\overline{\mathrm{Dev}}$ & $\mathrm{TC}$ & $\overline{\mathrm{Dev}}$ & & $=$ & $<$ \\
\hline R1A (12) & 4180.83 & -1.51 & 4128.48 & -0.24 & 4131.31 & -0.31 & 4113.89 & 0.12 & 4103.16 & 0.38 & 4118.70 & 0 & 0 \\
\hline R1B (12) & 1927.57 & -1.65 & 1902.19 & -0.31 & 1898.88 & -0.13 & 1896.83 & -0.03 & 1891.63 & 0.25 & 1896.35 & 0 & $1^{*}$ \\
\hline $\mathrm{R} 1 \mathrm{C}(12)$ & 1615.44 & -2.56 & 1582.18 & -0.45 & 1579.17 & -0.26 & 1578.12 & -0.19 & 1574.32 & 0.05 & 1575.09 & 1 & 0 \\
\hline $\mathrm{C} 1 \mathrm{~A}(9)$ & 7229.02 & -1.20 & 7143.35 & 0.00 & 7141.15 & 0.03 & 7139.96 & 0.05 & 7138.93 & 0.06 & 7143.35 & 2 & 0 \\
\hline C1B (9) & 2384.77 & -0.99 & 2361.78 & -0.02 & 2365.49 & -0.18 & 2359.82 & 0.06 & 2359.63 & 0.07 & 2361.29 & 2 & $1^{*}$ \\
\hline $\mathrm{C} 1 \mathrm{C}(9)$ & 1629.70 & -0.62 & 1621.09 & -0.09 & 1621.83 & -0.14 & 1618.91 & 0.04 & 1619.18 & 0.00 & 1619.18 & 6 & 0 \\
\hline $\mathrm{RC} 1 \mathrm{~A}(8)$ & 5117.96 & -3.49 & 4961.69 & -0.33 & 4948.53 & -0.07 & 4948.02 & -0.06 & 4915.10 & 0.61 & 4945.14 & 0 & 0 \\
\hline $\mathrm{RC} 1 \mathrm{~B}(8)$ & 2163.51 & -1.35 & 2142.65 & -0.37 & 2129.60 & 0.24 & 2136.73 & -0.09 & 2129.04 & 0.27 & 2134.74 & 0 & $2^{*}$ \\
\hline $\mathrm{RC} 1 \mathrm{C}(8)$ & 1784.51 & -1.36 & 1769.93 & -0.53 & 1758.29 & 0.13 & 1762.34 & -0.10 & 1752.19 & 0.48 & 1760.59 & 0 & 0 \\
\hline $\mathrm{R} 2 \mathrm{~A}(11)$ & 3568.97 & -9.06 & 3304.57 & -0.98 & 3310.70 & -1.17 & 3287.80 & -0.47 & 3267.31 & 0.16 & 3272.48 & 2 & $1^{*}$ \\
\hline R2B (11) & 1727.04 & -17.40 & 1498.97 & -1.88 & 1495.37 & -1.64 & 1487.09 & -1.08 & 1480.30 & -0.61 & $1471.27^{*}$ & 1 & $7^{*}$ \\
\hline $\mathrm{R} 2 \mathrm{C}(11)$ & 1436.22 & -15.30 & 1281.31 & -2.84 & 1257.65 & -0.94 & 1260.97 & -1.20 & 1237.79 & 0.66 & 1245.97 & 0 & 0 \\
\hline $\mathrm{C} 2 \mathrm{~A}(8)$ & 6267.75 & -9.07 & 5759.02 & -0.22 & 5797.38 & -0.89 & 5749.98 & -0.06 & 5760.29 & -0.24 & $5746.44^{*}$ & 4 & 0 \\
\hline C2B (8) & 1897.62 & -8.53 & 1754.07 & -0.32 & 1756.08 & -0.43 & 1748.99 & -0.03 & 1750.37 & -0.11 & $1748.52^{*}$ & 2 & $1^{*}$ \\
\hline $\mathrm{C} 2 \mathrm{C}(8)$ & 1276.29 & -4.78 & 1232.98 & -1.22 & 1223.86 & -0.47 & 1224.08 & -0.49 & 1221.17 & -0.25 & $1218.12^{*}$ & 4 & $2^{*}$ \\
\hline $\mathrm{RC} 2 \mathrm{~A}(8)$ & 4752.95 & -8.24 & 4406.28 & -0.34 & 4399.12 & -0.18 & 4388.88 & 0.05 & 4381.73 & 0.21 & 4391.16 & 0 & 0 \\
\hline $\mathrm{RC} 2 \mathrm{~B}(8)$ & 2156.11 & -15.40 & 1888.83 & -1.13 & 1899.20 & -1.68 & 1874.86 & -0.38 & 1877.84 & -0.54 & $1867.80^{*}$ & 0 & $2^{*}$ \\
\hline $\mathrm{RC} 2 \mathrm{C}(8)$ & 1828.95 & -19.50 & 1567.22 & -2.43 & 1562.19 & -2.10 & 1541.13 & -0.72 & 1545.29 & -0.99 & $1530.08^{*}$ & 0 & 0 \\
\hline $\operatorname{Min}(\%)$ & & -19.50 & & -2.84 & & -2.10 & & -1.20 & & -0.99 & & & \\
\hline Avg (\%) & & -6.78 & & -0.76 & & -0.57 & & -0.25 & & 0.03 & & & \\
\hline $\operatorname{Max}(\%)$ & & -0.62 & & 0.00 & & 0.24 & & 0.12 & & 0.66 & & & \\
\hline & & & & & & & & & & & & 24 & $17^{*}$ \\
\hline Runs & 1 & & 1 & & 3 & & 1 & & 10 & & & & \\
\hline Processor & P $600 \mathrm{M}$ & & PIV 1.5 & & Ath 2.60 & & PIV $3.4 \mathrm{G}$ & & Opt $2.2 \mathrm{G}$ & & $\mathrm{Xe} 2.6 \mathrm{GHz}$ & & \\
\hline Avg Time & 14.15 & & 20.00 & & 10.97 & & 16.67 & & 5.08 & & 4.83 & & \\
\hline
\end{tabular}

case performance is $0.94 \%$. The HEA outperforms all other algorithms in the literature for FD, including the UHGS of Vidal et al. (2014). 
Table 3: Average results for FD

\begin{tabular}{|c|c|c|c|c|c|c|c|c|c|}
\hline \multirow[t]{2}{*}{ Instance set } & \multicolumn{2}{|l|}{ MDA } & \multicolumn{2}{|l|}{$\overline{B P D R T}$} & \multicolumn{2}{|l|}{ UHGS } & \multirow{2}{*}{$\begin{array}{l}\text { HEA } \\
\text { TC }\end{array}$} & \multicolumn{2}{|c|}{ BKS } \\
\hline & TC & Dev & $\mathrm{TC}$ & Dev & TC & Dev & & $=$ & $<$ \\
\hline R1A (12) & 4068.59 & -0.67 & 4060.96 & -0.48 & 4031.28 & 0.25 & 4041.46 & 0 & 0 \\
\hline R1B (12) & 1854.60 & -0.82 & - & - & 1841.43 & -0.11 & 1839.39* & 0 & $4^{*}$ \\
\hline $\mathrm{R} 1 \mathrm{C}(12)$ & 1539.48 & -0.91 & 1539.90 & -0.93 & 1530.25 & -0.30 & $1525.56^{*}$ & 0 & $8^{*}$ \\
\hline C1A (9) & 7085.56 & -0.03 & 7085.91 & -0.04 & 7082.98 & 0.00 & 7082.98 & 9 & 0 \\
\hline C1B (9) & 2335.11 & -0.09 & - & - & 2332.89 & 0.00 & 2332.90 & 9 & 0 \\
\hline $\mathrm{C} 1 \mathrm{C}(9)$ & 1615.75 & -0.02 & 1615.40 & -0.01 & 1615.49 & -0.01 & $1615.38^{*}$ & 9 & 0 \\
\hline RC1A (8) & 4944.48 & -0.57 & 4935.52 & -0.38 & 4891.25 & 0.51 & 4916.41 & 0 & 0 \\
\hline RC1B (8) & 2121.62 & -0.87 & - & - & 2107.08 & -0.18 & $2103.21^{*}$ & 0 & $7^{*}$ \\
\hline $\mathrm{RC} 1 \mathrm{C}(8)$ & 1741.78 & -0.94 & 1749.66 & -1.40 & 1734.36 & -0.51 & $1725.44^{*}$ & 2 & $6^{*}$ \\
\hline R2A (11) & 3193.41 & -1.36 & 3180.59 & -0.96 & 3151.96 & -0.05 & $3150.29 *$ & 7 & $4^{*}$ \\
\hline $\mathrm{R} 2 \mathrm{~B}(11)$ & 1392.92 & -3.06 & - & - & 1351.905 & -0.02 & $1351.52^{*}$ & 4 & $2^{*}$ \\
\hline $\mathrm{R} 2 \mathrm{C}(11)$ & 1149.65 & -2.06 & 1149.11 & -2.01 & 1128.708 & -0.20 & $1126.42^{*}$ & 5 & $4^{*}$ \\
\hline C2A (8) & 5690.87 & -0.07 & 5689.40 & -0.04 & 5686.75 & 0.00 & 5686.75 & 8 & 0 \\
\hline C2B (8) & 1698.51 & -0.69 & - & - & 1686.75 & 0.00 & 1686.75 & 8 & 0 \\
\hline $\mathrm{C} 2 \mathrm{C}(8)$ & 1186.03 & -0.07 & 1185.70 & -0.04 & 1185.19 & 0.00 & 1185.19 & 8 & 0 \\
\hline $\mathrm{RC} 2 \mathrm{~A}(8)$ & 4241.33 & -0.73 & 4231.25 & -0.49 & 4210.10 & 0.00 & 4210.10 & 5 & $1^{*}$ \\
\hline $\mathrm{RC} 2 \mathrm{~B}(8)$ & 1704.13 & -1.04 & - & - & 1686.63 & -0.01 & $1686.47^{*}$ & 0 & $5^{*}$ \\
\hline $\mathrm{RC} 2 \mathrm{C}(8)$ & 1374.55 & -1.11 & 1385.32 & -1.91 & 1358.24 & 0.08 & 1359.33 & 1 & $3^{*}$ \\
\hline $\operatorname{Min}(\%)$ & & -4.30 & & -7.74 & & -1.49 & & & \\
\hline Avg (\%) & & -0.90 & & -0.74 & & -0.02 & & & \\
\hline $\operatorname{Max}(\%)$ & & 0.07 & & 0.10 & & 0.94 & & & \\
\hline All & & & & & & & & 75 & $44^{*}$ \\
\hline Runs & 3 & & 1 & & & & 10 & & \\
\hline Processor & Ath $2.6 \mathrm{C}$ & & Duo $2.4 \mathrm{G}$ & & Opt $2.2 \mathrm{G}$ & & Xe $2.6 \mathrm{G}$ & & \\
\hline Avg Time & 3.56 & & - & & 4.72 & & 4.56 & & \\
\hline
\end{tabular}

fleet mix composition and lower distribution costs than the other methods.

In summary, the HEA was able to find 41 BKS for 168 FT instances, where 17 are strictly better than those obtained by competing heuristics. As for FD, the algorithm has identified 119 BKS out of the 168 instances, 44 of which are strictly better than those obtained by previous heuristics. The results are even more striking for $\mathrm{HT}$, with $17 \mathrm{BKS}$ on the 24 instances, 14 of which are strictly better than those reported earlier. Overall, the HEA improves 75 BKS and matches 102 BKS out of 360 benchmark instances.

\section{Conclusions}

We have proposed a unified heuristic for four types of heterogeneous fleet vehicle routing problems with time windows. The first two are the Fleet Size and Mix Vehicle Routing Problem with Time Windows (F) and the Heterogeneous Fixed Fleet Vehicle Routing Problem with Time Windows $(\mathrm{H})$. Each of these two problems was solved under a time and a distance objective, yielding the four variants FT, FD, HT and HD. We have developed a unified hybrid evolutionary algorithm (HEA) capable of solving all variants without any modifica- 
Table 4: Results for HT

\begin{tabular}{|c|c|c|c|c|c|c|c|c|c|c|c|c|c|}
\hline \multirow[t]{2}{*}{ Instance set } & \multicolumn{3}{|l|}{$\mathrm{LSa}$} & \multicolumn{3}{|l|}{ ReVNTS } & \multicolumn{5}{|l|}{ HEA } & \multicolumn{2}{|c|}{ BKS } \\
\hline & Mix & $\mathrm{TC}$ & Dev & Mix & TC & Dev & $\mathrm{DC}$ & $\mathrm{VC}$ & Mix & TC & Time & $=$ & $<$ \\
\hline R101A & $A^{1} B^{11} C^{11} D^{1}$ & 5061 & -10.29 & $B^{10} C^{11} D^{1}$ & 4583.99 & 0.10 & 1998.76 & 2590 & $B^{10} C^{11} D^{1}$ & 4588.76 & 5.49 & 0 & 0 \\
\hline R102A & $A^{1} B^{4} C^{14} D^{2}$ & 5013 & -13.25 & $B^{3} C^{14} D^{2}$ & 4420.68 & 0.13 & 1736.54 & 2640 & $A^{1} B^{4} C^{13} D^{2}$ & $4376.54^{*}$ & 6.78 & 0 & $1 *$ \\
\hline R103A & $B^{7} C^{15}$ & 4772 & -13.57 & $B^{6} C^{15}$ & 4195.05 & 0.16 & 1621.71 & 2580 & $B^{6} C^{15}$ & 4201.71 & 7.45 & 0 & 0 \\
\hline $\mathrm{R} 104 \mathrm{~A}$ & $B^{9} C^{14}$ & 4455 & -10.61 & $B^{8} C^{14}$ & 4065.52 & -0.94 & 1487.69 & 2540 & $B^{9} C^{13}$ & $4027.69^{*}$ & 6.14 & 0 & $1 *$ \\
\hline C101A & $A^{1} B^{10}$ & 9272 & -5.02 & $B^{10}$ & 8828.93 & 0.00 & 828.93 & 8000 & $B^{10}$ & 8828.93 & 3.67 & 1 & 0 \\
\hline $\mathrm{C} 102 \mathrm{~A}$ & $A^{19}$ & 8433 & -17.89 & $A^{19}$ & 7137.79 & 0.21 & 1453.13 & 5700 & $A^{19}$ & 7153.13 & 4.12 & 0 & 0 \\
\hline C103A & $A^{19}$ & 8033 & -12.78 & $A^{19}$ & 7143.88 & -0.30 & 1422.57 & 5700 & $A^{19}$ & $7122.57^{*}$ & 3.45 & 0 & $1 *$ \\
\hline C104A & $A^{19}$ & 7384 & -4.25 & $A^{19}$ & 7104.96 & -0.30 & 1383.74 & 5700 & $A^{19}$ & $7083.74^{*}$ & 3.13 & 0 & $1 *$ \\
\hline RC101A & $A^{7} B^{7} C^{7}$ & 5687 & -7.99 & $A^{4} B^{7} C^{7}$ & 5279.92 & -0.26 & 1876.36 & 3390 & $A^{4} B^{7} C^{7}$ & $5266.36^{*}$ & 5.73 & 0 & $1 *$ \\
\hline $\mathrm{RC} 102 \mathrm{~A}$ & $A^{5} B^{6} C^{8}$ & 5649 & -10.77 & $A^{4} B^{5} C^{8}$ & 5149.95 & -0.99 & 1709.55 & 3390 & $A^{4} B^{5} C^{8}$ & $5099.55^{*}$ & 5.14 & 0 & $1 *$ \\
\hline $\mathrm{RC} 103 \mathrm{~A}$ & $A^{11} B^{2} C^{8}$ & 5419 & -8.58 & $A^{10} B^{2} C^{8}$ & 5002.41 & -0.22 & 1691.29 & 3300 & $A^{10} B^{2} C^{8}$ & $4991.29 *$ & 4.90 & 0 & $1 *$ \\
\hline $\mathrm{RC} 104 \mathrm{~A}$ & $A^{2} B^{13} C^{3} D^{1}$ & 5189 & -3.43 & $A^{2} B^{13} C^{3} D^{1}$ & 5024.25 & -0.15 & 1596.97 & 3420 & $A^{2} B^{13} C^{3} D^{1}$ & $5016.97 *$ & 5.21 & 0 & $1 *$ \\
\hline $\mathrm{R} 201 \mathrm{~A}$ & $A^{5}$ & 4593 & -21.43 & $A^{5}$ & 3779.12 & 0.09 & 1532.49 & 2250 & $A^{5}$ & 3782.49 & 7.45 & 0 & 0 \\
\hline R202A & $A^{5}$ & 4331 & -20.85 & $A^{5}$ & 3578.91 & 0.14 & 1333.92 & 2250 & $A^{5}$ & 3583.92 & 8.45 & 0 & 0 \\
\hline R203A & $A^{4} B^{1}$ & 4220 & -18.74 & $A^{4} B^{1}$ & 3582.54 & -0.81 & 1053.92 & 2500 & $A^{4} B^{1}$ & $3553.92^{*}$ & 7.12 & 0 & $1 *$ \\
\hline $\mathrm{R} 204 \mathrm{~A}$ & $A^{5}$ & 3849 & -24.89 & $A^{5}$ & 3143.68 & -2.01 & 831.80 & 2250 & $A^{5}$ & $3081.80 *$ & 6.99 & 0 & $1 *$ \\
\hline C201A & $A^{4} B^{1}$ & 6711 & -9.29 & $A^{4} B^{1}$ & 6140.64 & 0.00 & 740.64 & 5400 & $A^{4} B^{1}$ & 6140.64 & 4.89 & 1 & 0 \\
\hline C202A & $A^{1} C^{3}$ & 7720 & -1.26 & $A^{1} C^{3}$ & 7752.88 & -1.69 & 623.96 & 7000 & $A^{1} C^{3}$ & $7623.96^{*}$ & 4.26 & 0 & $1 *$ \\
\hline C203A & $C^{2} D^{1}$ & 7466 & -2.23 & $C^{2} D^{1}$ & 7303.37 & 0.00 & 603.37 & 6700 & $C^{2} D^{1}$ & 7303.37 & 4.37 & 1 & 0 \\
\hline C204A & $A^{5}$ & 6744 & -18.72 & $A^{5}$ & 5721.09 & -0.72 & 680.46 & 5000 & $A^{5}$ & $5680.46^{*}$ & 5.29 & 0 & $1 *$ \\
\hline $\mathrm{RC} 201 \mathrm{~A}$ & $C^{1} E^{3}$ & 5871 & -6.08 & $C^{1} E^{3}$ & 5523.15 & 0.21 & 1684.59 & 3850 & $C^{1} E^{3}$ & 5534.59 & 6.47 & 0 & 0 \\
\hline $\mathrm{RC} 202 \mathrm{~A}$ & $A^{1} C^{1} D^{1} E^{2}$ & 5945 & -15.43 & $A^{1} C^{1} D^{1} E^{2}$ & 5132.08 & 0.35 & 1450.23 & 3700 & $A^{1} C^{1} D^{1} E^{2}$ & 5150.23 & 6.35 & 0 & 0 \\
\hline RC203A & $A^{1} B^{1} C^{5}$ & 5790 & -29.47 & $A^{1} B^{1} C^{5}$ & 4508.27 & -0.81 & 1221.92 & 3250 & $A^{1} B^{1} C^{5}$ & $4471.92^{*}$ & 6.01 & 0 & $1 *$ \\
\hline $\mathrm{RC} 204 \mathrm{~A}$ & $A^{14} B^{2}$ & 4983 & -17.47 & $A^{14} B^{2}$ & 4252.87 & -0.26 & 1441.83 & 2800 & $A^{14} B^{2}$ & $4241.83^{*}$ & 5.87 & 0 & $1 *$ \\
\hline $\operatorname{Min}(\%)$ & & & -29.47 & & & -2.01 & & & & & & & \\
\hline Avg (\%) & & & -12.68 & & & -0.34 & & & & & & & \\
\hline $\operatorname{Max}(\%)$ & & & -1.26 & & & 0.35 & & & & & & & \\
\hline Total & & & & & & & & & & & & 3 & $14^{*}$ \\
\hline Runs & 3 & & & 1 & & & 10 & & & & & & \\
\hline Processor & P 233M & & & PIV $1.5 \mathrm{GHz}$ & & & $\mathrm{Xe} 2.6 \mathrm{GHz}$ & & & & & & \\
\hline Avg Time & - & & & 20.00 & & & 5.61 & & & & & & \\
\hline
\end{tabular}


Table 5: Results for HD

\begin{tabular}{|c|c|c|c|c|c|}
\hline \multirow[t]{2}{*}{ Instance set } & \multicolumn{5}{|l|}{ HEA } \\
\hline & $\overline{\mathrm{DC}}$ & $\mathrm{VC}$ & Mix & $\mathrm{TC}$ & Time \\
\hline R101A & 1765.41 & 2590 & $B^{10} C^{11} D^{1}$ & 4355.41 & 5.19 \\
\hline $\mathrm{R} 102 \mathrm{~A}$ & 1716.44 & 2640 & $B^{4} C^{13} D^{2}$ & 4356.44 & 6.24 \\
\hline R103A & 1500.16 & 2580 & $B^{6} C^{15}$ & 4080.16 & 6.57 \\
\hline R104A & 1434.72 & 2520 & $B^{7} C 14$ & 3954.72 & 5.89 \\
\hline C101A & 828.94 & 8000 & $B^{10}$ & 8828.94 & 4.25 \\
\hline C102A & 1380.17 & 5700 & $A^{19}$ & 7080.17 & 3.97 \\
\hline C103A & 1379.21 & 5700 & $A^{19}$ & 7079.21 & 3.99 \\
\hline C104A & 1375.06 & 5700 & $A^{19}$ & 7075.06 & 2.98 \\
\hline RC101A & 1772.28 & 3390 & $A^{4} B^{7} C^{7}$ & 5162.28 & 6.41 \\
\hline $\mathrm{RC} 102 \mathrm{~A}$ & 1598.05 & 3420 & $A^{2} B^{6} C^{8}$ & 5018.05 & 5.24 \\
\hline $\mathrm{RC} 103 \mathrm{~A}$ & 1626.55 & 3300 & $A^{10} B^{2} C^{8}$ & 4926.55 & 4.39 \\
\hline $\mathrm{RC} 104 \mathrm{~A}$ & 1575.91 & 3420 & $A^{2} B^{13} C^{3} D^{1}$ & 4995.91 & 4.88 \\
\hline $\mathrm{R} 201 \mathrm{~A}$ & 1198.76 & 2250 & $A^{5}$ & 3448.76 & 6.74 \\
\hline $\mathrm{R} 202 \mathrm{~A}$ & 1058.16 & 2250 & $A^{5}$ & 3308.16 & 8.13 \\
\hline $\mathrm{R} 203 \mathrm{~A}$ & 882.39 & 2500 & $A^{4} B^{1}$ & 3382.39 & 7.49 \\
\hline $\mathrm{R} 204 \mathrm{~A}$ & 768.14 & 2250 & $A^{5}$ & 3018.14 & 5.47 \\
\hline C201A & 682.38 & 5400 & $A^{4} B^{1}$ & 6082.38 & 4.21 \\
\hline $\mathrm{C} 202 \mathrm{~A}$ & 618.62 & 7000 & $A^{1} C^{3}$ & 7618.62 & 3.69 \\
\hline C203A & 603.37 & 6700 & $C^{2} D^{1}$ & 7303.37 & 3.67 \\
\hline C204A & 677.66 & 5000 & $A^{5}$ & 5677.66 & 5.11 \\
\hline $\mathrm{RC} 201 \mathrm{~A}$ & 1494.47 & 3850 & $C^{1} E^{3}$ & 5344.47 & 6.72 \\
\hline $\mathrm{RC} 202 \mathrm{~A}$ & 1156.02 & 3700 & $A^{1} C^{1} D^{1} E^{2}$ & 4856.02 & 6.48 \\
\hline $\mathrm{RC} 203 \mathrm{~A}$ & 996.25 & 3250 & $A^{1} B^{1} C^{5}$ & 4246.25 & 6.93 \\
\hline $\mathrm{RC} 204 \mathrm{~A}$ & 1395.32 & 2800 & $A^{14} B^{2}$ & 4195.32 & 6.17 \\
\hline Average & & & & 5224.77 & 5.45 \\
\hline Runs & 10 & & & & \\
\hline Processor & $\mathrm{Xe} 2.6 \mathrm{GHz}$ & & & & \\
\hline Avg Time & 5.45 & & & & \\
\hline
\end{tabular}


tion. This heuristic combines state-of-the-art metaheuristic principles such as heterogeneous adaptive large scale neighborhood search and population search. We have integrated within our HEA an innovative INTENSIFICATION strategy on elite solutions and we have developed a new diversification scheme based on the REGENERATION and the MutATION of solutions. We have also developed an advanced version of the SPLIT algorithm of Prins (2009) to determine the best fleet mix for a set of routes. Finally, we have introduced the new variant HD. Extensive computational experiments were carried out on benchmark instances. In the case of FT, our HEA clearly outperforms all previous algorithms except that of Vidal et al. (2014). It performs slightly worse on average, but is superior on instances which are less tight in terms of vehicle capacity. On the FD instances, our HEA outperforms the three existing algorithms. Overall, the HEA has identified 160 new best solutions out of 336 on the $\mathrm{F}$ instances, 61 of which are strictly better than previously known solutions. On the HT instances, our HEA outperforms the two existing algorithms and has identified 17 best known solutions out of 24, 14 of which are strictly better than previously found solutions. The HD instances are solved here for the first time. Overall, we have improved 75 solutions out of 360 instances, and we have matched 102 others. All instances were solved within a modest computational effort. Our algorithm is not only highly competitive, but it is also flexible in that it can solve four problem classes with the same parameter settings.

\section{Acknowledgement}

The authors gratefully acknowledge funding provided by the Southampton Business School of University of Southampton and by the Canadian Natural Sciences and Engineering Research Council under grants 39682-10 and 436014-2013. Thanks are due to a referee who provided valuable advice on a previous version of this paper.

\section{Appendix}

Table A.1 to A.6 present the detailed results on all benchmark instances for FT and FD. 


\section{References}

Baldacci, R., Battarra, M., D. Vigo. 2008. Routing a heterogeneous fleet of vehicles. In B. Golden, S. Raghavan, E. Wasil, eds. The Vehicle Routing Problem: Latest Advances and New Challenges. Springer, New York, 1-25.

Baldacci, R., A. Mingozzi. 2009. A unified exact method for solving different classes of vehicle routing problems. Mathematical Programming 120, 347-380.

Baldacci, R., Toth, P., D. Vigo. 2010. Exact algorithms for routing problems under vehicle capacity constraints. Annals of Operations Research 175, 213-245.

Belfiore, P., H. T. Y. Yoshizaki. 2009. Scatter search for a real-life heterogeneous fleet vehicle routing problem with time windows and split deliveries in Brazil. European Journal of Operational Research 199, 750-758.

Belfiore, P., H. T. Y. Yoshizaki. 2013. Heuristic methods for the fleet size and mix vehicle routing problem with time windows and split deliveries. Computers \& Industrial Engineering 64, 589-601.

Bettinelli, A., Ceselli, A., G. Righini. 2011. A branch-and-cut-and-price algorithm for the multi-depot heterogeneous vehicle routing problem with time windows. Transportation Research Part C: Emerging Technologies 19, 723-740.

Bräysy, O., Dullaert, W., Hasle, G., Mester, D., M. Gendreau. 2008. An effective multirestart deterministic annealing metaheuristic for the fleet size and mix vehicle routing problem with time windows. Transportation Science 42, 371-386.

Bräysy, O., Porkka, P. P., Dullaert, W., Repoussis, P. P., C. D. Tarantilis. 2009. A well scalable metaheuristic for the fleet size and mix vehicle-routing problem with time windows. Expert System with Applications 36, $8460-8475$.

Clarke, G., J. W. Wright. 1964. Scheduling of vehicles from a central depot to a number of delivery points. Operations Research 12, 568-581.

Cordeau, J.-F., Laporte, G., A. Mercier. 2001. A unified tabu search heuristic for vehicle routing problems with time windows. Journal of the Operational Research Society 52, 928-936.

Dell'Amico, M., Monaci, M., Pagani, C., D. Vigo. 2007. Heuristic approaches for the fleet size and mix vehicle routing problem with time windows. Transportation Science 41, 516-526.

Demir, E., Bektaş, T., G. Laporte. 2012. An adaptive large neighborhood search heuristic for the PollutionRouting Problem. European Journal of Operational Research 223, 346-359.

Dondo, R., J. Cerdá. 2007. A cluster-based optimization approach for the multi-depot heterogeneous fleet vehicle routing problem with time windows. European Journal of Operational Research 176, 1478-1507.

Dullaert, W., Janssens, G. K., Sörensen, K., B. Vernimmen. 2002. New heuristics for the fleet size and mix vehicle routing problem with time windows. Journal of the Operational Research Society 53, 1232-1238.

Golden, B. L., Assad, A. A., Levy, L., F. Gheysens. 1984. The fleet size and mix vehicle routing problem. 
Computers \& Operations Research 11, 49-66.

Hoff, A., Andersson, H., Christiansen, M., Hasle, G., A. Løkketangen. 2010. Industrial aspects and literature survey: fleet composition and routing. Computers 65 Operations Research 37, 2041-2061.

Kritikos, M.N., G. Ioannou. 2013. The heterogeneous fleet vehicle routing problem with overloads and time windows. International Journal of Production Economics 144, 68-75.

Koç, Ç., Bektaş, T., Jabali, O., Laporte, G. 2014. The fleet size and mix pollution-routing problem. Transportation Research Part B: Methodological 70, 239-254.

Liu, F. H., S. Y. Shen. 1999a. A method for vehicle routing problem with multiple vehicle types and times windows. Proceedings of the National Science Council, Republic of China, Part A: Physical Science and Engineering 23, 526-536.

Liu, F. H., S. Y. Shen. 1999b. The fleet size and mix vehicle routing problem with time windows. Journal of the Operational Research Society 50, 721-732.

Nagata, Y., Bräysy, O., W. Dullaert. 2010. A penalty-based edge assembly memetic algorithm for the vehicle routing problem with time windows. Computers $\&$ Operations Research 37, 724-737.

Paraskevopoulos, D. C., Repoussis, P. P., Tarantilis, C. D., Ioannou, G., G. P. Prastacos. 2008. A reactive variable neighbourhood tabu search for the heterogeneous fleet vehicle routing problem with time windows. Journal of Heuristics 14, 425-455.

Pisinger, D., S. Ropke. 2007. A general heuristic for vehicle routing problems. Computers E Operations Research 34, 2403-2435.

Prins, C. 2004. A simple and effective evolutionary algorithm for the vehicle routing problem. Computers $\&$ Operations Research 31, 1985-2002.

Prins, C. 2009. Two memetic algorithms for heterogeneous fleet vehicle routing problems. Engineering Applications of Artificial Intelligence 22, 916-928.

Repoussis, P. P., C. D. Tarantilis. 2010. Solving the fleet size and mix vehicle routing problem with time windows via adaptive memory programming. Transportation Research Part C: Emerging Technologies 18, 695-712.

Ropke, S., D. Pisinger. 2006a. An adaptive large neighborhood search heuristic for the pickup and delivery problem with time windows. Transportation Science 40, 455-472.

Ropke, S., D. Pisinger. 2006b. A unified heuristic for a large class of vehicle routing problems with backhauls. European Journal of Operational Research 171, 750-775.

Shaw, P., 1998. Using constraint programming and local search methods to solve vehicle routing problems. In: Proceedings of the 4th International Conference on Principles and Practice of Constraint Programming. (pp. 417-431). Springer, New York.

Solomon, M. M. 1987. Algorithms for the vehicle routing and scheduling problems with time window con- 
straints. Operations Research 35, 254-265.

Taillard, É.D. 1999. A heuristic column generation method for the heterogeneous fleet vehicle routing problem. RAIRO (Recherche Opérationnelle / Operations Research) 33, 1-14.

Toth, P., D. Vigo. 2002. Models, relaxations and exact approaches for the capacitated vehicle routing problem. Discrete Applied Mathematics 123, 487-512.

Toth, P. and Vigo, D., eds. (2014), Vehicle Routing: Problems, Methods, and Applications. MOS-SIAM Series on Optimization, Philadelphia.

Vidal, T., Crainic, T. G., Gendreau, M., Lahrichi, N., W. Rei. 2012. A hybrid genetic algorithm for multidepot and periodic vehicle routing problems. Operations Research 60, 611-624.

Vidal, T., Crainic, T. G., Gendreau, M., C. Prins. 2013. A hybrid genetic algorithm with adaptive diversity management for a large class of vehicle routing problems with time-windows, Computers $\& 3$ Operations Research 40, 475-489.

Vidal, T., Crainic, T. G., Gendreau, M., C. Prins. 2014. A unified solution framework for multi-attribute vehicle routing problems. European Journal of Operational Research 234, 658-673. 
Table A.1: Results for FT for cost structure $A$

\begin{tabular}{|c|c|c|c|c|c|c|c|c|c|c|c|c|c|}
\hline \multirow[t]{2}{*}{ Instance set } & \multicolumn{2}{|l|}{ ReVNTS } & \multicolumn{2}{|l|}{ MDA } & \multicolumn{2}{|l|}{ AMP } & \multicolumn{2}{|l|}{ UHGS } & \multicolumn{5}{|l|}{ HEA } \\
\hline & $\mathrm{TC}$ & Dev & $\mathrm{TC}$ & Dev & $\mathrm{TC}$ & Dev & $\mathrm{TC}$ & Dev & $\mathrm{DC}$ & $\mathrm{VC}$ & Mix & $\mathrm{TC}$ & Time \\
\hline R101A & 4539.99 & 0.04 & 4631.31 & -1.97 & 4536.4 & 0.12 & 4608.62 & -1.50 & 1951.70 & 2590 & $A^{1} B^{2} C$ & 4541.70 & 5.26 \\
\hline R102A & 375.70 & -0.47 & 4401.31 & -1.06 & 4348.92 & 0.14 & 4369.74 & -0.30 & 1775.10 & 2580 & $B^{6} C^{15}$ & 4355.10 & 5.87 \\
\hline R103A & 4120.63 & 0.26 & 4182.16 & -1.23 & 4119.04 & 0.30 & 4145.68 & -0.30 & 1551.23 & 2580 & $B^{6} C^{15}$ & 4131.23 & 4.19 \\
\hline R104A & 3992.65 & -0.01 & 3981.28 & 0.27 & 3986.35 & 0.14 & 3961.39 & 0.77 & 1302.10 & 2690 & $B^{5} C^{11} D$ & 3992.10 & 5.02 \\
\hline R105A & 4229.69 & 0.07 & 4236.84 & -0.10 & 4229.67 & 0.07 & 4209.84 & 0.54 & 1672.54 & 2560 & $B^{4} C^{16}$ & 4232.54 & 4.73 \\
\hline R106A & 4137.96 & 0.01 & 4118.48 & 0.48 & 4130.82 & 0.18 & 4109.08 & 0.71 & 1538.30 & 2600 & $B^{1} C^{18}$ & 4138.30 & 5.13 \\
\hline R107A & 4061.10 & -0.66 & 4035.96 & -0.04 & 4031.16 & 0.08 & 4007.87 & 0.66 & 1474.32 & 2560 & $B^{4} C^{16}$ & 4034.32 & 5.4 \\
\hline R108A & 3986.07 & -0.50 & 3970.26 & -0.10 & 3962.2 & 0.10 & 3934.48 & 0.80 & 1406.10 & 2560 & $B^{4} C^{16}$ & 3966.10 & 4.78 \\
\hline R109A & 4086.72 & -0.68 & 4060.17 & -0.03 & 4052.21 & 0.17 & 4020.75 & 0.94 & 1429.02 & 2630 & $C^{17} D^{1}$ & 4059.02 & 4.6 \\
\hline R110A & 30.85 & -0.86 & 3995.18 & 0.03 & 3999.09 & -0.07 & 3965.88 & 0.76 & 1436.31 & 2560 & $B^{4} C^{1} 6$ & 3996.31 & 4.17 \\
\hline R111A & 4018.80 & 0.03 & 4017.81 & 0.06 & 4016.19 & 0.10 & 3985.68 & 0.86 & 1460.10 & 2560 & $B^{4} C^{13} D^{2}$ & 4020.10 & 4.98 \\
\hline R112A & 3961.63 & -0.10 & 3947.30 & 0.26 & 3954.65 & 0.07 & 3918.88 & 0.98 & 1397.60 & 2560 & $B^{4} C^{16}$ & 3957.60 & 5.78 \\
\hline C101A & 7226.51 & 0.00 & 7226.51 & 0.00 & 7226.51 & 0.00 & 7226.51 & 0.00 & 1526.51 & 5700 & $A^{19}$ & 7226.51 & 2.97 \\
\hline C102A & 7137.79 & 0.11 & 7119.35 & 0.37 & 7137.79 & 0.11 & 7119.35 & 0.37 & 445.65 & 5700 & $A^{19}$ & 7145.65 & 3.10 \\
\hline C103A & 7143.88 & 0.00 & 7107.01 & 0.52 & 7141.03 & 0.04 & 7102.86 & 0.57 & 443.88 & 5700 & $A^{19}$ & 7143.88 & 2.70 \\
\hline C104A & 7104.96 & -0.31 & 7081.50 & 0.02 & 7086.70 & -0.05 & 7081.51 & 0.02 & 382.92 & 5700 & $A^{19}$ & 7082.92 & 2.01 \\
\hline C105A & 7171.48 & 0.05 & 7199.36 & -0.34 & 7169.08 & 0.08 & 7196.06 & -0.3 & 475.00 & 5700 & $A^{19}$ & 7175.00 & 2.45 \\
\hline $6 \mathrm{~A}$ & 7157.13 & 0.09 & 7180.03 & -0.23 & 7157.13 & 0.09 & 7176.68 & -0.20 & 1463.32 & 5700 & $A^{19}$ & 7163.32 & 3.01 \\
\hline C107A & 7135.43 & 0.07 & 7149.17 & -0.13 & 7135.38 & 0.07 & 7144.49 & -0.10 & 1440.20 & 5700 & $A^{19}$ & 7140.20 & 2.78 \\
\hline C108A & 7115.71 & 0.07 & 7115.81 & 0.07 & 7113.57 & 0.10 & 7111.23 & 0.14 & 420.98 & 5700 & $A^{19}$ & 712 & 2.45 \\
\hline C109A & 7095.55 & -0.05 & 7094.65 & -0.04 & 7092.49 & -0.01 & 7091.66 & 0.00 & 391.66 & 5700 & $A^{19}$ & 7091.66 & 2.37 \\
\hline $\mathrm{RC}$ & 5253.86 & -0.35 & 5253.97 & -0.35 & 5237.19 & -0.03 & 5217.90 & 0.33 & 815.42 & 3420 & $A^{2} B$ & 5235.42 & 4.97 \\
\hline $\mathrm{RC}$ & 5053.48 & -0.47 & 5059.58 & -0.59 & 62 & -0 & 5018.47 & 0.22 & 639.69 & 3390 & $A^{4} B^{3} C^{9}$ & 5029.69 & 5.64 \\
\hline $\mathrm{A}$ & 92.80 & -0.47 & 4868.94 & 0.02 & 58 & -0.32 & 4822.21 & 0.98 & 480.00 & 3390 & $A^{4} L$ & 00 & 5.14 \\
\hline$A$ & 83.31 & -0.29 & 4762.85 & 0.14 & 61.28 & 0.17 & 4737.00 & 0.68 & 1289.30 & 3480 & $A^{3} B$ & 30 & 4.97 \\
\hline $\mathrm{R}$ & 12.91 & 0.10 & 5119.80 & -0.03 & 10.86 & 0.14 & 5097.35 & 0.41 & 1788.10 & 3330 & $A^{3} B$ & 10 & 5.32 \\
\hline $\mathrm{A}$ & 7.98 & -0.79 & 4960.78 & -0.04 & 66.27 & -0.15 & 4935.91 & 0.46 & 568.62 & 3390 & $A^{4} B^{9} C^{6}$ & 62 & 6.01 \\
\hline A & 62.67 & -0.78 & 4828.17 & -0.06 & 4819.91 & 0.11 & 4783.08 & 0.87 & 1405.21 & 3420 & $A 4 B$ & 4825.21 & 5.37 \\
\hline RC108A & 36.50 & 0.38 & 4734.15 & 0.43 & 4749.44 & 0.11 & 4708.85 & 0.97 & 1244.77 & 3510 & $A^{1} B^{2} C$ & 4754.77 & 4.71 \\
\hline R201A & 79.12 & -0.50 & 3922.00 & -4.3 & 3753.42 & 0.19 & 3782.88 & -0.6 & 1510.43 & 2250 & $A^{5}$ & 3760.43 & 8.97 \\
\hline R202A & 78.91 & -0.70 & 3610.38 & -1.58 & 551.12 & 0.09 & 3540.03 & 0.40 & 1304.20 & 2250 & $A^{5}$ & 3554.20 & 9.98 \\
\hline R203A & 34.08 & -0.56 & 3350.18 & -1.05 & 336.60 & -0.64 & 3311.35 & 0.13 & 1065.50 & 2250 & $A^{5}$ & 3315.50 & 8.76 \\
\hline R204A & 43.68 & -2.20 & 3390.14 & -10.20 & 103.84 & -0.91 & 3075.95 & 0.00 & 25.95 & 2250 & $A^{5}$ & 3075.95 & 7.98 \\
\hline R205A & 71.47 & -1.12 & 3465.81 & -3.95 & 3367.90 & -1.01 & 3334.27 & 0.00 & 1084.27 & 2250 & $A^{5}$ & 3334.27 & 8.45 \\
\hline $\mathrm{R} 206 \mathrm{~A}$ & 72.79 & -0.29 & 3268.36 & -0.15 & 264.70 & -0.04 & 3242.40 & 0.64 & 1013.40 & 2250 & $A^{5}$ & 3263.40 & 8.17 \\
\hline R207A & 13.60 & -1.94 & 3231.26 & -2.51 & 58.69 & -0.20 & 3145.08 & 0.23 & 902.29 & 2250 & $A^{5}$ & 3152.29 & 9.29 \\
\hline R208A & 64.76 & -1.58 & 3063.10 & -1.52 & 56.45 & -1.30 & 3017.52 & -0.01 & 767.12 & 2250 & $A^{5}$ & $3017.12^{*}$ & 8.51 \\
\hline R209A & 91.63 & 0.08 & 3192.95 & 0.04 & 94.74 & -0.01 & 3183.36 & 0.34 & 44.28 & 2250 & $A^{5}$ & 3194.28 & 9.37 \\
\hline R210A & 38.75 & -0.89 & 3375.38 & -2.00 & 325.28 & -0.48 & 3287.66 & 0.65 & 059.26 & 2250 & $A^{5}$ & 3309.26 & 8.79 \\
\hline $\mathrm{R} 211 \mathrm{~A}$ & 61.47 & -1.35 & 3042.48 & -0.73 & 3053.08 & -1.08 & 3019.93 & 0.02 & 770.56 & 2250 & $A^{5}$ & 3020.56 & 7.99 \\
\hline C201A & 5820.78 & 0.16 & 5891.45 & -1.05 & 5820.78 & 0.16 & 5878.54 & -0.80 & 830.20 & 5000 & $A^{5}$ & 5830.20 & 5.00 \\
\hline C202A & 5779.59 & -0.05 & 5850.26 & -1.27 & 5783.76 & -0.12 & 5776.88 & 0.00 & 776.88 & 5000 & $A^{5}$ & 5776.88 & 5.17 \\
\hline C203A & 5750.58 & -0.15 & 5741.90 & -0.00 & 5736.94 & 0.09 & 5741.12 & 0.00 & 741.89 & 5000 & $A^{5}$ & 5741.12 & 4.76 \\
\hline C204A & 721.09 & -0.72 & 5691.51 & -0.19 & 5718.49 & -0.67 & 5680.46 & 0.00 & 680.46 & 5000 & $A^{5}$ & 5680.46 & 4.21 \\
\hline C205A & 5750.53 & 0.02 & 5786.71 & -0.61 & 5747.67 & 0.06 & 5781.15 & -0.50 & 751.40 & 5000 & $A^{5}$ & 5751.40 & 6.79 \\
\hline C206A & 5757.93 & -0.29 & 5795.15 & -0.94 & 5738.09 & 0.06 & 5767.70 & -0.50 & 741.30 & 5000 & $A^{5}$ & 5741.30 & 4.3 \\
\hline C207A & 5723.91 & 0.02 & 5743.52 & -0.32 & 5721.16 & 0.07 & 5731.44 & -0.10 & 725.10 & 5000 & $A^{5}$ & 5725.10 & 4.17 \\
\hline C208A & 67.78 & -0.75 & 5884.20 & -2.78 & 5732.95 & -0.14 & 5725.03 & 0.00 & 725.03 & 5000 & $A^{5}$ & 5725.03 & 5.21 \\
\hline $\mathrm{RC} 201 \mathrm{~A}$ & 26.22 & -0.39 & 4740.21 & -0.69 & 4701.88 & 0.13 & 4737.59 & -0.60 & 2007.80 & 2700 & $A^{18}$ & 4707.80 & 4.50 \\
\hline $\mathrm{RC} 202 \mathrm{~A}$ & 4518.49 & 0.02 & 4522.36 & -0.07 & 4509.11 & 0.23 & 4487.48 & 0.71 & 1619.40 & 2900 & $A^{10} B^{4}$ & 4519.40 & 4.67 \\
\hline $\mathrm{RC} 203 \mathrm{~A}$ & 327.57 & -0.20 & 4312.52 & 0.15 & 4313.42 & 0.13 & 4305.49 & 0.32 & 1469.10 & 2850 & $A^{12} B^{3}$ & 4319.10 & 5.27 \\
\hline $\mathrm{RC} 204 \mathrm{~A}$ & 66.73 & -0.26 & 4141.04 & 0.35 & 4157.32 & -0.04 & 4137.93 & 0.43 & 1005.77 & 3150 & $A^{2} B^{5} C^{2}$ & 4155.77 & 5.19 \\
\hline $\mathrm{RC} 205 \mathrm{~A}$ & 4645.41 & -1.08 & 4652.57 & -1.24 & 4585.20 & 0.23 & 4615.04 & -0.40 & 1795.67 & 2800 & $A^{14} B^{2}$ & 4595.67 & 6.89 \\
\hline $\mathrm{RC} 206 \mathrm{~A}$ & 4416.41 & 0.40 & 4431.64 & 0.06 & 4427.73 & 0.15 & 4405.16 & 0.66 & 1584.30 & 2850 & $A^{9} B^{3} C^{1}$ & 4434.30 & 5.03 \\
\hline $\mathrm{RC} 207 \mathrm{~A}$ & 4338.94 & -0.53 & 4310.11 & 0.13 & 4313.07 & 0.07 & 4290.14 & 0.60 & 1215.90 & 3100 & $A^{4} B^{7}$ & 4315.90 & 6.27 \\
\hline $\mathrm{RC} 208 \mathrm{~A}$ & 4109.90 & -0.70 & 4091.92 & -0.26 & 4103.31 & -0.54 & 4075.04 & 0.16 & 1031.37 & 3050 & $A^{5} B^{5} C^{1}$ & 4081.37 & 5.17 \\
\hline
\end{tabular}


Table A.2: Results for FT for cost structure $B$

\begin{tabular}{|c|c|c|c|c|c|c|c|c|c|c|c|c|c|}
\hline \multirow[t]{2}{*}{ Instance set } & \multicolumn{2}{|l|}{ ReVNTS } & \multicolumn{2}{|l|}{ MDA } & \multicolumn{2}{|l|}{ AMP } & \multicolumn{2}{|l|}{ UHGS } & \multicolumn{5}{|l|}{ HEA } \\
\hline & $\mathrm{TC}$ & Dev & $\mathrm{TC}$ & $\overline{\text { Dev }}$ & $\mathrm{TC}$ & $\overline{\text { Dev }}$ & $\mathrm{TC}$ & Dev & $\mathrm{DC}$ & $\mathrm{VC}$ & Mix & $\mathrm{TC}$ & Time \\
\hline R101B & 2421.19 & 0.16 & 2486.76 & -2.54 & 2421.19 & 0.16 & 2421.19 & 0.16 & 1849.10 & 576 & $A^{1} B^{4} C^{9} D^{5}$ & 2425.10 & 3.78 \\
\hline R102B & 2219.03 & -0.30 & 2227.48 & -0.68 & 2209.50 & 0.13 & 2209.50 & 0.13 & 1608.37 & 604 & $A^{2} B^{1} C^{6} D^{8}$ & 2212.37 & 3.97 \\
\hline R103B & 1955.57 & -0.18 & 1938.93 & 0.67 & 1953.50 & -0.08 & 1938.93 & 0.67 & 1313.99 & 638 & $A^{1} B^{1} C^{4} D^{6} E^{2}$ & 1951.99 & 4.28 \\
\hline R104B & 1732.26 & -1.01 & 1714.73 & 0.01 & 1713.36 & 0.09 & 1713.36 & 0.09 & 1026.86 & 688 & $A^{1} C^{1} D^{5} E^{4}$ & 1714.86 & 4.01 \\
\hline R105B & 2030.83 & -0.29 & 2027.98 & -0.15 & 2030.83 & -0.29 & 2027.98 & -0.15 & 1436.91 & 588 & $B^{3} C^{5} D^{8}$ & $2024.91^{*}$ & 3.68 \\
\hline R106B & 1924.03 & -0.1 & 1919.03 & 0.16 & 1919.02 & 0.16 & 1919.02 & 0.16 & 1338.10 & 584 & $B^{1} C^{6} D^{8}$ & 1922.10 & 4.19 \\
\hline R107B & 1781.01 & 0.12 & 1789.58 & -0.36 & 1780.52 & 0.15 & 1780.52 & 0.15 & 1127.20 & 656 & $C^{2} D^{8} E^{2}$ & 1783.20 & 5.30 \\
\hline R108B & 1667.51 & -0.36 & 1649.24 & 0.74 & 1665.78 & -0.25 & 1649.24 & 0.74 & 983.58 & 678 & $C^{1} D^{5} E^{4}$ & 1661.58 & 4.78 \\
\hline R109B & 1844.99 & -0.87 & 1828.63 & 0.03 & 1840.54 & -0.63 & 1828.63 & 0.03 & 1185.10 & 644 & $B^{1} C^{1} D^{10} E^{1}$ & 1829.10 & 4.91 \\
\hline R110B & 1792.75 & -0.78 & 1774.46 & 0.24 & 1788.18 & -0.53 & 1774.46 & 0.24 & 1178.80 & 600 & $B^{1} C^{3} D^{10}$ & 1778.80 & 5.21 \\
\hline R111B & 1780.03 & -0.27 & 1769.71 & 0.31 & 1772.51 & 0.15 & 1769.71 & 0.31 & 1141.24 & 634 & $C^{3} D^{7} E^{2}$ & 1775.24 & 4.78 \\
\hline R112B & 1677.13 & -0.01 & 1669.78 & 0.43 & 1667.00 & 0.60 & 1667.00 & 0.60 & 1071.00 & 606 & $C^{2} D^{11}$ & 1677.00 & 6.21 \\
\hline C101B & 2417.52 & 0.00 & 2417.52 & 0.00 & 2417.52 & 0.00 & 2417.52 & 0.00 & 977.52 & 1440 & $A^{8} B^{6}$ & 2417.52 & 1.99 \\
\hline C102B & 2350.54 & 0.00 & 2350.54 & 0.00 & 2350.54 & 0.00 & 2350.54 & 0.00 & 930.54 & 1420 & $A^{5} B^{7}$ & 2350.54 & 2.45 \\
\hline C103B & 2349.42 & -0.18 & 2353.64 & -0.36 & 2347.99 & -0.11 & 2347.99 & -0.11 & 925.31 & 1420 & $A^{5} B^{7}$ & $2345.31^{*}$ & 3.47 \\
\hline C104B & 2332.94 & -0.10 & 2328.62 & 0.08 & 2325.78 & 0.21 & 2325.78 & 0.21 & 950.59 & 1380 & $A^{7} B^{6}$ & 2330.59 & 3.09 \\
\hline C105B & 2374.01 & 0.10 & 2373.53 & 0.12 & 2375.04 & 0.06 & 2373.53 & 0.12 & 956.45 & 1420 & $A^{5} B^{7}$ & 2376.45 & 3.06 \\
\hline C106B & 2381.14 & 0.22 & 2404.56 & -0.76 & 2381.14 & 0.22 & 2381.14 & 0.22 & 966.43 & 1420 & $A^{5} B^{7}$ & 2386.43 & 2.95 \\
\hline C107B & 2357.52 & 0.06 & 2370 & -0.47 & 2357.67 & 0.06 & 2357.52 & 0.06 & 939.00 & 1420 & $A^{5} B^{7}$ & 2359.00 & 2.45 \\
\hline C108B & 2346.38 & 0.08 & 2346.38 & 0.08 & 2346.38 & 0.08 & 2346.38 & 0.08 & 968.15 & 1380 & $A^{7} B^{6}$ & 2348.15 & 2.79 \\
\hline C109B & 2346.58 & -0.38 & 2339.89 & -0.10 & 2336.29 & 0.06 & 2336.29 & 0.06 & 957.6 & 1380 & $A^{7} B^{6}$ & 2337.60 & 2.56 \\
\hline RC101B & 2469.50 & -0.22 & 2462.60 & 0.06 & 2464.66 & -0.02 & 2462.60 & 0.06 & 1732.19 & 732 & $A^{1} B^{4} C^{10}$ & 2464.19 & 4.47 \\
\hline $\mathrm{RC} 102 \mathrm{~B}$ & 2277.79 & -0.32 & 2263.45 & 0.31 & 2272.68 & -0.10 & 2263.45 & 0.31 & 1538.43 & 732 & $A^{1} B^{3} C^{9} D^{1}$ & 2270.43 & 4.12 \\
\hline RC103B & 2057.55 & -0.80 & 2035.62 & 0.27 & 2041.24 & -0.00 & 2035.62 & 0.27 & 1291.20 & 750 & $B^{1} C^{9} D^{2}$ & 2041.20 & 3.98 \\
\hline RC104B & 1914.93 & 0.38 & 1905.06 & 0.90 & 1916.85 & 0.28 & 1905.06 & 0.90 & 1172.27 & 750 & $B^{1} C^{6} D^{4}$ & 1922.27 & 4.21 \\
\hline RC105B & 2337.93 & -0.44 & 2308.59 & 0.82 & 2325.99 & 0.07 & 2308.59 & 0.82 & 1625.70 & 702 & $A^{1} B^{7} C^{8}$ & 2327.70 & 4.56 \\
\hline $\mathrm{RC} 106 \mathrm{~B}$ & 2168.44 & -0.99 & 2149.56 & -0.11 & 2160.45 & -0.62 & 2149.56 & -0.11 & 1415.14 & 732 & $A^{1} B^{2} C^{8} D^{2}$ & $2147.14^{*}$ & 4.21 \\
\hline RC107B & 2008.39 & -0.62 & 2000.77 & -0.23 & 2003.26 & -0.36 & 2000.77 & -0.23 & 1264.09 & 732 & $A^{1} B^{2} C^{5} D^{4}$ & $1996.09^{*}$ & 4.19 \\
\hline RC108B & 1906.69 & 0.12 & 1910.83 & -0.10 & 1908.72 & 0.01 & 1906.69 & 0.12 & 1176.89 & 732 & $A^{1} B^{1} C^{7} D^{3}$ & 1908.89 & 3.11 \\
\hline R201B & 1965.10 & -0.45 & 2002.53 & -2.37 & 1953.42 & 0.14 & 1953.42 & 0.14 & 1456.21 & 500 & $A^{4} B^{1}$ & 1956.21 & 6.21 \\
\hline R202B & 1765.09 & -0.72 & 1790.38 & -2.17 & 1751.12 & 0.07 & 1751.12 & 0.07 & 1302.4 & 450 & $A^{5}$ & 1752.40 & 8.00 \\
\hline R203B & 1535.08 & -1.31 & 1541.19 & -1.72 & 1536.60 & -1.41 & 1535.08 & -1.31 & 1065.17 & 450 & $A^{5}$ & $1515.17^{*}$ & 5.78 \\
\hline R204B & 1306.72 & -2.12 & 1284.33 & -0.37 & 1303.84 & -1.90 & 1284.33 & -0.37 & 829.57 & 450 & $A^{5}$ & $1279.57^{*}$ & 6.89 \\
\hline R205B & 1575.75 & -1.70 & 1563.62 & -0.92 & 1560.07 & -0.69 & 1560.07 & -0.69 & 1099.39 & 450 & $A^{5}$ & $1549.39^{*}$ & 6.49 \\
\hline $\mathrm{R} 206 \mathrm{~B}$ & 1477.34 & -1.86 & 1464.53 & -0.98 & 1464.70 & -0.99 & 1464.53 & -0.98 & 1000.37 & 450 & $A^{5}$ & $1450.37^{*}$ & 5.21 \\
\hline R207B & 1386.84 & -2.04 & 1380.41 & -1.56 & 1358.69 & 0.04 & 1358.69 & 0.04 & 909.18 & 450 & $A^{5}$ & 1359.18 & 6.31 \\
\hline R208B & 1261.09 & -3.34 & 1244.74 & -2.00 & 1256.45 & -2.96 & 1244.74 & -2.00 & 770.36 & 450 & $A^{5}$ & $1220.36^{*}$ & 5.47 \\
\hline R209B & 1418.51 & -2.37 & 1431.37 & -3.30 & 1394.74 & -0.66 & 1394.74 & -0.66 & 935.65 & 450 & $A^{5}$ & $1385.65^{*}$ & 7.14 \\
\hline R210B & 1529.04 & -2.23 & 1516.66 & -1.40 & 1525.28 & -1.97 & 1516.66 & -1.40 & 1045.75 & 450 & $A^{5}$ & $1495.75^{*}$ & 6.93 \\
\hline $\mathrm{R} 211 \mathrm{~B}$ & 1268.14 & -3.95 & 1255.06 & -2.88 & 1253.08 & -2.72 & 1219.93 & 0.00 & 770.56 & 450 & $A^{5}$ & 1219.93 & 7.45 \\
\hline C201B & 1816.14 & 0.25 & 1820.64 & 0.00 & 1816.14 & 0.25 & 1820.64 & 0.00 & 740.64 & 1080 & $A^{4} B^{1}$ & 1820.64 & 3.11 \\
\hline C202B & 1768.51 & 0.09 & 1795.40 & -1.43 & 1768.51 & 0.09 & 1768.51 & 0.09 & 690.10 & 1080 & $A^{2} B^{1} C^{1}$ & 1770.10 & 4.58 \\
\hline C203B & 1744.28 & -0.61 & 1733.63 & 0.00 & 1734.82 & -0.07 & 1733.63 & 0.00 & 653.63 & 1080 & $A^{2} B^{1} C^{1}$ & 1733.63 & 3.19 \\
\hline C204B & 1736.09 & -3.31 & 1708.69 & -1.68 & 1716.18 & -2.13 & 1680.46 & 0.00 & 680.46 & 1000 & $A^{5}$ & 1680.46 & 3.17 \\
\hline C205B & 1747.68 & 0.50 & 1782.74 & -1.49 & 1747.68 & 0.50 & 1778.30 & -1.24 & 716.54 & 1040 & $A^{1} B^{3}$ & 1756.54 & 5.21 \\
\hline C206B & 1756.93 & 0.92 & 1772.87 & 0.02 & 1756.01 & 0.97 & 1767.70 & 0.31 & 733.17 & 1040 & $A^{1} B^{3}$ & 1773.17 & 3.46 \\
\hline C207B & 1732.20 & -0.16 & 1729.49 & -0.01 & 1729.39 & -0.00 & 1729.49 & -0.01 & 689.39 & 1040 & $A^{1} B^{3}$ & $1729.39^{*}$ & 2.97 \\
\hline C208B & 1730.72 & -0.38 & 1724.2 & 0.00 & 1723.2 & 0.06 & 1724.20 & 0.00 & 684.20 & 1040 & $A^{1} B^{3}$ & 1724.20 & 3.13 \\
\hline $\mathrm{RC} 201 \mathrm{~B}$ & 2231.69 & 0.19 & 2343.79 & -4.83 & 2230.54 & 0.24 & 2329.59 & -4.19 & 1615.90 & 620 & $A^{4} B^{4} C^{2}$ & 2235.90 & 4.17 \\
\hline RC202B & 2002.62 & 0.96 & 2091.53 & -3.44 & 2022.54 & -0.03 & 2057.66 & -1.76 & 1392.00 & 630 & $A^{3} B^{3} C^{3}$ & $2022.00^{*}$ & 5.47 \\
\hline RC203B & 1843.72 & -0.18 & 1852.74 & -0.67 & 1841.26 & -0.05 & 1824.54 & 0.86 & 1190.40 & 650 & $B^{3} C^{4}$ & 1840.40 & 5.12 \\
\hline RC204B & 1611.28 & -3.57 & 1565.31 & -0.62 & 1575.18 & -1.25 & 1555.75 & -0.01 & 885.74 & 670 & $B^{1} C^{4} D^{1}$ & $1555.74^{*}$ & 4.98 \\
\hline RC205B & 2195.62 & -1.23 & 2195.75 & -1.23 & 2166.62 & 0.11 & 2174.74 & -0.26 & 1529.00 & 640 & $A^{2} B^{2} C^{4}$ & 2169.00 & 6.47 \\
\hline $\mathrm{RC} 206 \mathrm{~B}$ & 1887.23 & 0.60 & 1923.56 & -1.31 & 1893.13 & 0.29 & 1883.08 & 0.82 & 1218.70 & 680 & $B^{5} C^{1} D^{1}$ & 1898.70 & 4.14 \\
\hline RC207B & 1780.72 & -2.93 & 1745.85 & -0.92 & 1743.23 & -0.76 & 1714.14 & 0.92 & 1080.00 & 650 & $B^{3} C^{4}$ & 1730.00 & 5.14 \\
\hline RC208B & 1557.74 & -4.50 & 1488.19 & 0.16 & 1526.78 & -2.42 & 1483.20 & 0.50 & 830.64 & 660 & $C^{6}$ & 1490.64 & 4.43 \\
\hline
\end{tabular}


Table A.3: Results for FT for cost structure $C$

\begin{tabular}{|c|c|c|c|c|c|c|c|c|c|c|c|c|c|}
\hline \multirow[t]{2}{*}{ Instance set } & \multicolumn{2}{|l|}{ ReVNTS } & \multicolumn{2}{|l|}{ MDA } & \multicolumn{2}{|l|}{ AMP } & \multicolumn{2}{|l|}{ UHGS } & \multicolumn{5}{|l|}{ HEA } \\
\hline & TC & Dev & TC & Dev & $\mathrm{TC}$ & Dev & $\mathrm{TC}$ & Dev & $\mathrm{DC}$ & $\mathrm{VC}$ & Mix & $\mathrm{TC}$ & Time \\
\hline R101C & 2134.90 & 0.11 & 2199.78 & -2.93 & 2134.90 & 0.11 & 2199.79 & -2.93 & 1840.20 & 297 & $A^{1} B^{2} C^{9} D^{6}$ & 2137.20 & 3.14 \\
\hline $\mathrm{R} 102 \mathrm{C}$ & 1913.37 & 0.08 & 1925.55 & -0.56 & 1913.37 & 0.08 & 1925.56 & -0.56 & 1599.87 & 315 & $A^{2} B^{3} C^{4} D^{7} E^{1}$ & 1914.87 & 6.21 \\
\hline R103C & 1633.62 & -0.77 & 1609.94 & 0.69 & 1631.47 & -0.63 & 1615.38 & 0.36 & 1310.20 & 311 & $A^{1} C^{4} D^{8} E^{1}$ & 1621.20 & 3.24 \\
\hline $\mathrm{R} 104 \mathrm{C}$ & 1382.82 & -0.52 & 1370.84 & 0.35 & 1377.81 & -0.16 & 1363.26 & 0.90 & 1025.60 & 350 & $D^{8} E^{3}$ & 1375.60 & 4.47 \\
\hline $\mathrm{R} 105 \mathrm{C}$ & 1729.57 & -0.44 & 1722.05 & 0.00 & 1729.57 & -0.44 & 1722.05 & 0.00 & 1403.05 & 319 & $B^{2} C^{2} D^{11}$ & 1722.05 & 3.17 \\
\hline $\mathrm{R} 106 \mathrm{C}$ & 1607.96 & 0.15 & 1602.87 & 0.47 & 1607.96 & 0.15 & 1599.04 & 0.71 & 1285.40 & 325 & $A^{1} C^{5} D^{6} E^{2}$ & 1610.40 & 4.08 \\
\hline $\mathrm{R} 107 \mathrm{C}$ & 1455.09 & -0.05 & 1456.02 & -0.12 & 1452.52 & 0.12 & 1442.97 & 0.78 & 1126.30 & 328 & $C^{2} D^{8} E^{2}$ & 1454.30 & 3.51 \\
\hline $\mathrm{R} 108 \mathrm{C}$ & 1331.54 & -0.12 & 1336.28 & -0.48 & 1330.28 & -0.03 & 1321.68 & 0.62 & 979.92 & 350 & $D^{6} E^{4}$ & 1329.92 & 5.33 \\
\hline R109C & 1525.65 & -1.23 & 1507.77 & -0.04 & 1519.37 & -0.81 & 1505.59 & 0.10 & 1185.10 & 322 & $B^{1} C^{1} D^{10} E^{1}$ & 1507.10 & 4.73 \\
\hline $\mathrm{R} 110 \mathrm{C}$ & 1463.91 & -0.89 & 1446.41 & 0.32 & 1457.43 & -0.44 & 1443.92 & 0.49 & 1109.06 & 342 & $C^{3} D^{4} E^{4}$ & 1451.06 & 5.46 \\
\hline $\mathrm{R} 111 \mathrm{C}$ & 1451.92 & -1.09 & 1447.88 & -0.80 & 1443.34 & -0.49 & 1423.47 & 0.89 & 1098.32 & 338 & $B^{1} D^{9} E^{2}$ & 1436.32 & 6.14 \\
\hline $\mathrm{R} 112 \mathrm{C}$ & 1355.78 & -1.09 & 1335.41 & 0.42 & 1339.44 & 0.12 & 1329.07 & 0.90 & 988.10 & 353 & $C^{2} D^{5} E^{4}$ & 1341.10 & 4.17 \\
\hline $\mathrm{C} 101 \mathrm{C}$ & 1628.94 & 0.00 & 1628.31 & 0.04 & 1628.94 & 0.00 & 1628.94 & 0.00 & 828.94 & 800 & $B^{10}$ & 1628.94 & 1.97 \\
\hline $\mathrm{C} 102 \mathrm{C}$ & 1610.96 & 0.00 & 1610.96 & 0.00 & 1610.96 & 0.00 & 1610.96 & 0.00 & 860.96 & 750 & $A^{1} B^{9}$ & 1610.96 & 2.53 \\
\hline $\mathrm{C} 103 \mathrm{C}$ & 1611.14 & -0.25 & 1619.68 & -0.78 & 1607.14 & 0.00 & 1607.14 & 0.00 & 857.14 & 750 & $A^{1} B^{9}$ & 1607.14 & 3.79 \\
\hline $\mathrm{C} 104 \mathrm{C}$ & 1610.07 & -0.68 & 1613.96 & -0.92 & 1598.50 & 0.04 & 1599.90 & -0.04 & 869.21 & 730 & $A^{3} B^{8}$ & 1599.21 & 2.89 \\
\hline $\mathrm{C} 105 \mathrm{C}$ & 1628.94 & 0.00 & 1628.38 & 0.03 & 1628.94 & 0.00 & 1628.94 & 0.00 & 828.94 & 800 & $B^{10}$ & 1628.94 & 1.97 \\
\hline $\mathrm{C} 106 \mathrm{C}$ & 1628.94 & 0.00 & 1628.94 & 0.00 & 1628.94 & 0.00 & 1628.94 & 0.00 & 828.94 & 800 & $B^{10}$ & 1628.94 & 2.01 \\
\hline $\mathrm{C} 107 \mathrm{C}$ & 1628.94 & 0.00 & 1628.38 & 0.03 & 1628.94 & 0.00 & 1628.94 & 0.00 & 828.94 & 800 & $B^{10}$ & 1628.94 & 1.99 \\
\hline $\mathrm{C} 108 \mathrm{C}$ & 1622.89 & 0.13 & 1622.89 & 0.13 & 1622.89 & 0.13 & 1622.89 & 0.13 & 825 & 800 & $B^{10}$ & 1625.00 & 2.45 \\
\hline $\mathrm{C} 109 \mathrm{C}$ & 1619.02 & -0.03 & 1614.99 & 0.22 & 1614.99 & 0.22 & 1615.93 & 0.17 & 888.61 & 730 & $A^{3} B^{8}$ & 1618.61 & 3.54 \\
\hline $\mathrm{RC} 101 \mathrm{C}$ & 2089.37 & 0.13 & 2084.48 & 0.36 & 2089.37 & 0.13 & 2082.95 & 0.44 & 1702.10 & 390 & $B^{7} C^{5} D^{3}$ & 2092.10 & 4.54 \\
\hline $\mathrm{RC} 102 \mathrm{C}$ & 1918.96 & -0.90 & 1895.92 & 0.31 & 1906.68 & -0.25 & 1895.05 & 0.36 & 1529.89 & 372 & $A^{2} B^{2} C^{8} D^{2}$ & 1901.89 & 4.19 \\
\hline $\mathrm{RC} 103 \mathrm{C}$ & 1674.50 & -0.83 & 1660.62 & 0.00 & 1666.24 & -0.33 & 1650.30 & 0.63 & 1300.7 & 360 & $C^{12}$ & 1660.70 & 3.56 \\
\hline $\mathrm{RC} 104 \mathrm{C}$ & 1543.55 & -0.19 & 1537.09 & 0.23 & 1540.13 & 0.03 & 1526.04 & 0.95 & 1159.60 & 381 & $A^{1} C^{5} D^{5}$ & 1540.60 & 3.47 \\
\hline $\mathrm{RC} 105 \mathrm{C}$ & 1972.57 & -0.84 & 1957.52 & -0.07 & 1953.99 & 0.11 & 1957.14 & -0.05 & 1584.09 & 372 & $A^{2} B^{2} C^{8} D^{2}$ & 1956.09 & 4.16 \\
\hline $\mathrm{RC} 106 \mathrm{C}$ & 1793.12 & -0.71 & 1776.08 & 0.25 & 1787.69 & -0.41 & 1774.94 & 0.31 & 1393.45 & 387 & $A^{2} B^{1} C^{6} D^{4}$ & 1780.45 & 3.49 \\
\hline $\mathrm{RC} 107 \mathrm{C}$ & 1635.65 & -0.95 & 1614.04 & 0.39 & 1622.90 & -0.16 & 1607.11 & 0.81 & 1245.30 & 375 & $B^{3} C^{5} D^{4}$ & 1620.30 & 3.07 \\
\hline $\mathrm{RC} 108 \mathrm{C}$ & 1531.69 & 0.06 & 1535.14 & -0.17 & 1531.69 & 0.06 & 1523.96 & 0.56 & 1157.60 & 375 & $B^{2} C^{6} D^{4}$ & 1532.60 & 3.56 \\
\hline $\mathrm{R} 201 \mathrm{C}$ & 1745.39 & -0.82 & 1729.92 & 0.07 & 1728.42 & 0.16 & 1716.02 & 0.88 & 1461.20 & 270 & $A^{6}$ & 1731.20 & 6.78 \\
\hline $\mathrm{R} 202 \mathrm{C}$ & 1537.33 & -0.50 & 1537.35 & -0.50 & 1527.92 & 0.12 & 1515.96 & 0.90 & 1304.70 & 225 & $A^{5}$ & 1529.70 & 8.14 \\
\hline R203C & 1338.42 & -3.22 & 1308.70 & -0.92 & 1311.60 & -1.15 & 1286.35 & 0.80 & 1071.72 & 225 & $A^{5}$ & 1296.72 & 6.50 \\
\hline $\mathrm{R} 204 \mathrm{C}$ & 1080.66 & -2.64 & 1062.46 & -0.91 & 1085.71 & -3.12 & 1050.95 & 0.19 & 802.90 & 250 & $A^{5}$ & 1052.90 & 7.89 \\
\hline $\mathrm{R} 205 \mathrm{C}$ & 1350.12 & -2.66 & 1311.84 & 0.26 & 1335.07 & -1.51 & 1309.27 & 0.45 & 1090.20 & 225 & $A^{5}$ & 1315.20 & 6.71 \\
\hline $\mathrm{R} 206 \mathrm{C}$ & 1254.67 & -2.26 & 1251.51 & -2.00 & 1239.70 & -1.04 & 1216.35 & 0.86 & 1001.93 & 225 & $A^{5}$ & 1226.93 & 6.59 \\
\hline $\mathrm{R} 207 \mathrm{C}$ & 1186.05 & -5.38 & 1149.23 & -2.11 & 1139.61 & -1.25 & 1120.08 & 0.48 & 900.50 & 225 & $A^{5}$ & 1125.50 & 6.98 \\
\hline $\mathrm{R} 208 \mathrm{C}$ & 1022.31 & -2.44 & 1009.26 & -1.13 & 1022.11 & -2.42 & 992.12 & 0.59 & 772.97 & 225 & $A^{5}$ & 997.97 & 5.87 \\
\hline R209C & 1233.07 & -5.91 & 1178.45 & -1.21 & 1171.41 & -0.61 & 1155.79 & 0.73 & 939.31 & 225 & $A^{4} B^{1}$ & 1164.31 & 7.14 \\
\hline R210C & 1284.72 & -1.18 & 1289.35 & -1.55 & 1281.08 & -0.90 & 1257.89 & 0.93 & 1019.70 & 250 & $A^{4} B^{1}$ & 1269.70 & 6.14 \\
\hline $\mathrm{R} 211 \mathrm{C}$ & 1061.70 & -6.64 & 1013.84 & -1.83 & 1028.08 & -3.26 & 994.93 & 0.07 & 770.58 & 225 & $A^{5}$ & 995.58 & 6.17 \\
\hline $\mathrm{C} 201 \mathrm{C}$ & 1269.41 & -1.47 & 1269.41 & -1.47 & 1269.41 & -1.47 & 1269.41 & -1.47 & 650.97 & 600 & $A^{2} C^{2}$ & $1250.97^{*}$ & 2.97 \\
\hline $\mathrm{C} 202 \mathrm{C}$ & 1252.24 & -0.92 & 1242.66 & -0.15 & 1244.54 & -0.30 & 1239.54 & 0.11 & 700.86 & 540 & $A^{2} B^{1} C^{1}$ & 1240.86 & 3.54 \\
\hline $\mathrm{C} 203 \mathrm{C}$ & 1228.13 & -2.89 & 1193.63 & 0.00 & 1203.42 & -0.82 & 1193.63 & 0.00 & 653.63 & 540 & $A^{2} B^{1} C^{1}$ & 1193.63 & 3.14 \\
\hline $\mathrm{C} 204 \mathrm{C}$ & 1207.03 & -2.59 & 1176.52 & 0.00 & 1188.18 & -0.99 & 1176.52 & 0.00 & 636.52 & 540 & $A^{2} B^{1} C^{1}$ & 1176.52 & 3.67 \\
\hline $\mathrm{C} 205 \mathrm{C}$ & 1245.51 & -0.44 & 1245.62 & -0.45 & 1239.60 & 0.04 & 1238.30 & 0.15 & 640.1 & 600 & $A^{2} B^{2}$ & 1240.10 & 4.29 \\
\hline $\mathrm{C} 206 \mathrm{C}$ & 1229.63 & -0.03 & 1245.05 & -1.29 & 1229.23 & 0.00 & 1238.30 & -0.74 & 629.23 & 600 & $A^{2} C^{2}$ & 1229.23 & 4.38 \\
\hline $\mathrm{C} 207 \mathrm{C}$ & 1221.16 & -0.97 & 1215.42 & -0.49 & 1213.07 & -0.30 & 1209.49 & -0.01 & 689.48 & 520 & $A^{2} B^{1} C^{1}$ & $1209.48^{*}$ & 3.56 \\
\hline $\mathrm{C} 208 \mathrm{C}$ & 1210.72 & -0.54 & 1204.20 & 0.00 & 1205.18 & -0.08 & 1204.20 & 0.00 & 684.2 & 520 & $A^{1} B^{3}$ & 1204.20 & 3.01 \\
\hline $\mathrm{RC} 201 \mathrm{C}$ & 1957.60 & -2.07 & 2004.53 & -4.52 & 1915.42 & 0.13 & 1996.79 & -4.11 & 1577.90 & 340 & $A^{3} B^{3} C^{2} D^{1}$ & 1917.90 & 4.65 \\
\hline $\mathrm{RC} 202 \mathrm{C}$ & 1699.48 & -1.16 & 1766.52 & -5.15 & 1677.62 & 0.14 & 1732.66 & -3.13 & 1355.00 & 325 & $A^{1} B^{5} C^{1} D^{1}$ & 1680.00 & 6.10 \\
\hline RC203C & 1510.13 & -0.66 & 1517.98 & -1.19 & 1504.35 & -0.28 & 1496.11 & 0.27 & 1160.20 & 340 & $A^{2} B^{1} C^{3} E^{1}$ & 1500.20 & 6.27 \\
\hline $\mathrm{RC} 204 \mathrm{C}$ & 1256.91 & -2.84 & 1238.66 & -1.35 & 1241.45 & -1.58 & 1220.75 & 0.12 & 887.16 & 335 & $B^{1} C^{4} E^{1}$ & 1222.16 & 5.47 \\
\hline $\mathrm{RC} 205 \mathrm{C}$ & 1901.71 & -4.32 & 1854.22 & -1.71 & 1822.07 & 0.05 & 1844.74 & -1.19 & 1453 & 370 & $B^{2} C^{4} D^{1}$ & 1823.00 & 5.29 \\
\hline $\mathrm{RC} 206 \mathrm{C}$ & 1598.84 & -2.21 & 1590.22 & -1.66 & 1586.61 & -1.43 & 1553.65 & 0.68 & 1224.3 & 340 & $B^{5} C^{1} E^{1}$ & 1564.30 & 4.70 \\
\hline $\mathrm{RC} 207 \mathrm{C}$ & 1431.65 & -3.61 & 1396.16 & -1.05 & 1406.26 & -1.78 & 1377.52 & 0.30 & 1026.71 & 355 & $C^{3} D^{1} E^{1}$ & 1381.71 & 5.67 \\
\hline $\mathrm{RC} 208 \mathrm{C}$ & 1181.47 & -2.61 & 1145.84 & 0.48 & 1175.23 & -2.07 & 1140.10 & 0.98 & 821.40 & 330 & $C^{6}$ & 1151.40 & 5.17 \\
\hline
\end{tabular}


Table A.4: Results for FD for cost structure $A$

\begin{tabular}{|c|c|c|c|c|c|c|c|c|c|c|c|}
\hline \multirow[t]{2}{*}{ Instance set } & \multicolumn{2}{|l|}{ MDA } & \multicolumn{2}{|l|}{ BPDRT } & \multicolumn{2}{|l|}{ UHGS } & \multicolumn{5}{|l|}{ HEA } \\
\hline & $\mathrm{TC}$ & Dev & $\mathrm{TC}$ & Dev & $\mathrm{TC}$ & Dev & DC & $\mathrm{VC}$ & Mix & TC & Time \\
\hline R101A & 4349.80 & -0.75 & 4342.72 & -0.58 & 4314.36 & 0.07 & 1787.52 & 2530 & $A^{1} B^{10} C^{12}$ & 4317.52 & 4.14 \\
\hline R102A & 4196.46 & -0.54 & 4189.21 & -0.37 & 4166.28 & 0.18 & 1623.84 & 2550 & $A^{1} B^{5} C^{15}$ & 4173.84 & 5.98 \\
\hline R103A & 4052.85 & -0.53 & 4051.62 & -0.50 & 4027.36 & 0.10 & 1401.40 & 2630 & $B^{1} C^{18}$ & 4031.40 & 5.21 \\
\hline R104A & 3978.48 & -0.81 & 3972.65 & -0.66 & 3936.40 & 0.25 & 1276.44 & 2670 & $B^{3} C^{15} D^{1}$ & 3946.44 & 4.12 \\
\hline R105A & 4161.72 & -0.67 & 4152.50 & -0.45 & 4122.50 & 0.28 & 1574.06 & 2560 & $A^{1} B^{5} C^{15}$ & 4134.06 & 6.01 \\
\hline R106A & 4095.20 & -0.87 & 4085.30 & -0.62 & 4048.59 & 0.28 & 1500.05 & 2560 & $B^{4} C^{16}$ & 4060.05 & 5.12 \\
\hline R107A & 4006.61 & -0.54 & 3996.74 & -0.29 & 3970.51 & 0.37 & 1395.12 & 2590 & $B^{3} C^{15} D^{1}$ & 3985.12 & 4.78 \\
\hline R108A & 3961.38 & -0.73 & 3949.50 & -0.43 & 3928.12 & 0.11 & 1342.60 & 2590 & $B^{3} C^{15} D^{1}$ & 3932.60 & 6.54 \\
\hline R109A & 4048.29 & -0.58 & 4035.89 & -0.27 & 4015.71 & 0.23 & 1464.83 & 2560 & $B^{4} C^{16}$ & 4024.83 & 6.12 \\
\hline R110A & 3997.88 & -0.61 & 3991.63 & -0.46 & 3961.68 & 0.30 & 1373.51 & 2600 & $B^{1} C^{18}$ & 3973.51 & 5.21 \\
\hline R111A & 4011.63 & -0.59 & 4009.61 & -0.54 & 3964.99 & 0.58 & 1368.00 & 2620 & $B^{3} C^{15} D^{1}$ & 3988.00 & 5.12 \\
\hline R112A & 3962.73 & -0.83 & 3954.19 & -0.61 & 3918.88 & 0.29 & 1300.19 & 2630 & $C^{17} D^{1}$ & 3930.19 & 4.71 \\
\hline C101A & 7098.04 & -0.06 & 7097.93 & -0.06 & 7093.45 & 0.00 & 1393.45 & 5700 & $A^{19}$ & 7093.45 & 2.47 \\
\hline C102A & 7086.11 & -0.08 & 7085.47 & -0.07 & 7080.17 & 0.00 & 1380.17 & 5700 & $A^{19}$ & 7080.17 & 2.65 \\
\hline C103A & 7080.35 & -0.02 & 7080.41 & -0.02 & 7079.21 & 0.00 & 1379.21 & 5700 & $A^{19}$ & 7079.21 & 2.01 \\
\hline C104A & 7076.90 & -0.03 & 7075.06 & 0.00 & 7075.06 & 0.00 & 1375.06 & 5700 & $A^{19}$ & 7075.06 & 1.97 \\
\hline C105A & 7096.19 & -0.04 & 7096.22 & -0.04 & 7093.45 & 0.00 & 1393.45 & 5700 & $A^{19}$ & 7093.45 & 2.65 \\
\hline C106A & 7086.91 & -0.04 & 7088.35 & -0.06 & 7083.87 & 0.00 & 1383.87 & 5700 & $A^{19}$ & 7083.87 & 2.17 \\
\hline C107A & 7084.92 & -0.00 & 7090.91 & -0.09 & 7084.61 & 0.00 & 1384.61 & 5700 & $A^{19}$ & 7084.61 & 2.39 \\
\hline C108A & 7082.49 & -0.04 & 7081.18 & -0.02 & 7079.66 & 0.00 & 1379.66 & 5700 & $A^{19}$ & 7079.66 & 1.97 \\
\hline C109A & 7078.13 & -0.01 & 7077.68 & -0.01 & 7077.30 & 0.00 & 1377.30 & 5700 & $A^{19}$ & 7077.30 & 2.19 \\
\hline RC101A & 5180.74 & -0.14 & 5168.23 & 0.10 & 5150.86 & 0.44 & 1843.47 & 3330 & $A^{3} B^{13} C^{4}$ & 5173.47 & 5.14 \\
\hline $\mathrm{RC} 102 \mathrm{~A}$ & 5029.59 & -0.21 & 5025.22 & -0.13 & 4987.24 & 0.63 & 1658.83 & 3360 & $A^{6} B^{6} C^{7}$ & 5018.83 & 4.26 \\
\hline $\mathrm{RC} 103 \mathrm{~A}$ & 4895.57 & -0.94 & 4888.53 & -0.79 & 4804.61 & 0.94 & 1430.20 & 3420 & $A^{2} B^{6} C^{8}$ & 4850.20 & 6.47 \\
\hline RC104A & 4760.56 & -0.74 & 4747.38 & -0.47 & 4717.63 & 0.16 & 1395.40 & 3330 & $A^{3} B^{2} C^{8} D^{1}$ & 4725.40 & 5.29 \\
\hline $\mathrm{RC} 105 \mathrm{~A}$ & 5060.37 & -0.23 & 5068.54 & -0.39 & 5035.35 & 0.27 & 1748.86 & 3300 & $A^{5} B^{8} C^{6}$ & 5048.86 & 4.78 \\
\hline $\mathrm{RC} 106 \mathrm{~A}$ & 4997.86 & -0.68 & 4972.11 & -0.16 & 4936.74 & 0.55 & 1514.13 & 3450 & $B^{7} C^{8}$ & 4964.13 & 5.29 \\
\hline $\mathrm{RC} 107 \mathrm{~A}$ & 4865.76 & -0.83 & 4861.04 & -0.73 & 4788.69 & 0.76 & 1435.60 & 3390 & $A^{4} B^{5} C^{8}$ & 4825.60 & 4.17 \\
\hline RC108A & 4765.37 & -0.86 & 4753.12 & -0.60 & 4708.85 & 0.34 & 1334.79 & 3390 & $A^{4} B^{2} C^{8} D^{1}$ & 4724.79 & 4.63 \\
\hline R201A & 3484.95 & -1.11 & 3530.24 & -2.42 & 3446.78 & 0.00 & 1196.78 & 2250 & $A^{5}$ & 3446.78 & 6.13 \\
\hline R202A & 3335.95 & -1.17 & 3335.61 & -1.16 & 3308.16 & -0.33 & 1047.42 & 2250 & $A^{5}$ & $3297.42^{*}$ & 7.46 \\
\hline R203A & 3173.95 & -1.05 & 3164.03 & -0.73 & 3141.09 & 0.00 & 891.09 & 2250 & $A^{5}$ & 3141.09 & 6.14 \\
\hline R204A & 3065.15 & -1.56 & 3029.83 & -0.39 & 3018.14 & 0.00 & 768.14 & 2250 & $A^{5}$ & 3018.14 & 6.28 \\
\hline R205A & 3277.69 & -1.82 & 3261.19 & -1.31 & 3218.97 & 0.00 & 968.97 & 2250 & $A^{5}$ & 3218.97 & 6.38 \\
\hline R206A & 3173.30 & -0.86 & 3165.85 & -0.62 & 3146.34 & 0.00 & 896.34 & 2250 & $A^{5}$ & 3146.34 & 8.14 \\
\hline R207A & 3136.47 & -1.92 & 3102.79 & -0.83 & 3077.58 & -0.01 & 827.36 & 2250 & $A^{5}$ & $3077.36^{*}$ & 6.47 \\
\hline R208A & 3050.00 & -1.76 & 3009.13 & -0.40 & 2997.24 & 0.00 & 747.25 & 2250 & $A^{5}$ & 2997.25 & 6.34 \\
\hline R209A & 3155.73 & -1.16 & 3155.60 & -1.16 & 3122.42 & -0.09 & 869.56 & 2250 & $A^{5}$ & 3119.56* & 4.99 \\
\hline R210A & 3219.23 & -1.54 & 3206.23 & -1.13 & 3174.85 & -0.14 & 920.41 & 2250 & $A^{5}$ & $3170.41 *$ & 5.47 \\
\hline R211A & 3055.04 & -1.16 & 3026.02 & -0.20 & 3019.93 & 0.00 & 769.93 & 2250 & $A^{5}$ & 3019.93 & 7.93 \\
\hline C201A & 5701.45 & -0.11 & 5700.87 & -0.10 & 5695.02 & 0.00 & 695.02 & 5000 & $A^{5}$ & 5695.02 & 3.46 \\
\hline C202A & 5689.70 & -0.08 & 5689.70 & -0.08 & 5685.24 & 0.00 & 685.24 & 5000 & $A^{5}$ & 5685.24 & 3.17 \\
\hline C203A & 5685.82 & -0.08 & 5681.55 & 0.00 & 5681.55 & 0.00 & 681.55 & 5000 & $A^{5}$ & 5681.55 & 4.29 \\
\hline C204A & 5690.30 & -0.22 & 5677.69 & 0.00 & 5677.66 & 0.00 & 677.67 & 5000 & $A^{5}$ & 5677.66 & 3.97 \\
\hline C205A & 5691.70 & -0.01 & 5691.70 & -0.01 & 5691.36 & 0.00 & 691.36 & 5000 & $A^{5}$ & 5691.36 & 3.46 \\
\hline C206A & 5691.70 & -0.04 & 5691.70 & -0.04 & 5689.32 & 0.00 & 689.32 & 5000 & $A^{5}$ & 5689.32 & 2.97 \\
\hline C207A & 5689.82 & -0.04 & 5692.36 & -0.09 & 5687.35 & 0.00 & 687.35 & 5000 & $A^{5}$ & 5687.35 & 4.10 \\
\hline C208A & 5686.50 & 0.00 & 5689.59 & -0.05 & 5686.50 & 0.00 & 686.50 & 5000 & $A^{5}$ & 5686.50 & 3.56 \\
\hline RC201A & 4407.68 & -0.71 & 4404.07 & -0.62 & 4374.09 & 0.06 & 1476.82 & 2900 & $A^{10} B^{4}$ & 4376.82 & 5.14 \\
\hline $\mathrm{RC} 202 \mathrm{~A}$ & 4277.67 & -0.78 & 4266.96 & -0.53 & 4244.63 & 0.00 & 1294.63 & 2950 & $A^{8} B^{5}$ & 4244.63 & 4.26 \\
\hline RC203A & 4204.85 & -0.83 & 4189.94 & -0.47 & 4170.17 & 0.00 & 1120.17 & 3050 & $A^{6} B^{3} C^{2}$ & 4170.17 & 6.14 \\
\hline $\mathrm{RC} 204 \mathrm{~A}$ & 4109.86 & -0.56 & 4098.34 & -0.27 & 4087.11 & 0.00 & 937.112 & 3150 & $A^{5} B^{2} C^{3}$ & 4087.11 & 5.47 \\
\hline RC205A & 4329.96 & -0.84 & 4304.52 & -0.25 & 4291.93 & 0.04 & 1343.73 & 2950 & $A^{8} B^{5}$ & 4293.73 & 4.19 \\
\hline $\mathrm{RC} 206 \mathrm{~A}$ & 4272.08 & -0.48 & 4272.82 & -0.49 & 4251.88 & 0.00 & 1251.88 & 3000 & $A^{6} B^{6}$ & 4251.88 & 4.27 \\
\hline RC207A & 4232.81 & -1.20 & 4219.52 & -0.89 & 4185.98 & -0.08 & 1182.44 & 3000 & $A^{6} B^{6}$ & $4182.44^{*}$ & 5.64 \\
\hline RC208A & 4095.71 & -0.51 & 4093.83 & -0.46 & 4075.04 & 0.00 & 975.04 & 3100 & $A^{4} B^{4} C^{2}$ & 4075.04 & 5.31 \\
\hline
\end{tabular}


Table A.5: Results for FD for cost structure $B$

\begin{tabular}{|c|c|c|c|c|c|c|c|c|c|c|c|}
\hline \multirow[t]{2}{*}{ Instance set } & \multicolumn{2}{|l|}{ MDA } & \multicolumn{2}{|c|}{ BPDRT } & \multicolumn{2}{|l|}{ UHGS } & \multicolumn{5}{|l|}{ HEA } \\
\hline & TC & Dev & $\mathrm{TC}$ & Dev & $\mathrm{TC}$ & Dev & $\mathrm{DC}$ & $\mathrm{VC}$ & Mix & TC & Time \\
\hline R101B & 2226.94 & -0.20 & - & - & 2228.67 & -0.27 & 1664.56 & 558 & $B^{5} C^{13} D^{2}$ & $2222.56^{*}$ & 4.27 \\
\hline $\mathrm{R} 102 \mathrm{~B}$ & 2071.90 & -1.16 & - & - & 2073.63 & -1.25 & 1476.12 & 572 & $A^{1} B^{2} C^{10} D^{5}$ & $2048.12^{*}$ & 3.28 \\
\hline R103B & 1857.22 & -0.08 & - & - & 1853.66 & 0.11 & 1249.74 & 606 & $A^{1} C^{7} D^{6} E^{1}$ & 1855.74 & 5.27 \\
\hline R104B & 1707.31 & -1.24 & - & - & 1683.33 & 0.18 & 1026.42 & 660 & $A^{1} C^{1} D^{10} E^{1}$ & 1686.42 & 5.09 \\
\hline R105B & 1995.07 & -0.71 & - & - & 1988.86 & -0.40 & 1390.96 & 590 & $C^{10} D^{6}$ & $1980.96^{*}$ & 3.37 \\
\hline R106B & 1903.95 & -0.72 & - & - & 1888.31 & 0.10 & 1290.28 & 600 & $C^{9} D^{5} E^{1}$ & 1890.28 & 4.19 \\
\hline R107B & 1766.18 & -0.81 & - & - & 1753.35 & -0.08 & 1140.02 & 612 & $C^{4} D^{8} E^{1}$ & $1752.02^{*}$ & 5.26 \\
\hline R108B & 1666.89 & -1.06 & - & - & 1647.88 & 0.09 & 983.37 & 666 & $B^{1} C^{1} D^{8} E^{1}$ & 1649.37 & 3.97 \\
\hline R109B & 1833.54 & -0.79 & - & - & 1818.15 & 0.05 & 1209.10 & 610 & $B^{1} C^{4} D^{8} E^{1}$ & 1819.10 & 3.99 \\
\hline R110B & 1781.74 & -1.12 & - & - & 1758.64 & 0.19 & 1161.96 & 600 & $C^{2} D^{11}$ & 1761.96 & 5.47 \\
\hline R111B & 1768.74 & -1.47 & - & - & 1740.86 & 0.13 & 1121.16 & 622 & $C^{4} D^{8} E^{1}$ & 1743.16 & 5.69 \\
\hline R112B & 1675.76 & -0.76 & - & - & 1661.85 & 0.07 & 1029.09 & 634 & $C^{1} D^{10} E^{1}$ & 1663.09 & 5.01 \\
\hline C101B & 2340.98 & -0.04 & - & - & 2340.15 & 0.00 & 960.15 & 1380 & $A^{7} B^{6}$ & 2340.15 & 2.98 \\
\hline C102B & 2326.53 & -0.04 & - & - & 2325.70 & 0.00 & 945.70 & 1380 & $A^{7} B^{6}$ & 2325.70 & 2.73 \\
\hline C103B & 2325.61 & -0.04 & - & - & 2324.60 & 0.00 & 944.60 & 1380 & $A^{7} B^{6}$ & 2324.60 & 3.64 \\
\hline C104B & 2318.04 & 0.00 & - & - & 2318.04 & 0.00 & 938.04 & 1380 & $A^{7} B^{6}$ & 2318.04 & 2.98 \\
\hline C105B & 2344.64 & -0.19 & - & - & 2340.15 & 0.00 & 960.15 & 1380 & $A^{7} B^{6}$ & 2340 & 2.71 \\
\hline C106B & 2345.85 & -0.24 & - & - & 2340.15 & 0.00 & 960.15 & 1380 & $A^{7} B^{6}$ & 2340 & 3.19 \\
\hline C107B & 2345.60 & -0.23 & - & - & 2340.15 & 0.00 & 960.15 & 1380 & $A^{7} B^{6}$ & 234 & 2.94 \\
\hline C108B & 2340.17 & -0.07 & - & - & 2338.58 & 0.00 & 58.58 & 1380 & $A^{7} B^{6}$ & 233 & 3.88 \\
\hline C109B & 2328.55 & 0.00 & - & - & 2328.55 & 0.00 & 948.55 & 1380 & $A^{7} B^{6}$ & 2328 & 3.12 \\
\hline RC101B & 2417.16 & -0.40 & - & - & 2412.71 & -0.22 & 1693.43 & 714 & $A^{2} B^{7} C$ & 2407 & 3.46 \\
\hline RC102B & 2234.47 & -0.69 & - & - & 2213.92 & 0.24 & 1487.23 & 732 & $A^{2} B^{7} C^{5} D 2$ & 2219 & 5.14 \\
\hline RC103B & 2025.74 & -0.51 & - & - & 2016.28 & -0.04 & 1295.55 & 720 & $B^{1} C^{10} D^{1}$ & 2015 & 3.69 \\
\hline RC104B & 1912.65 & -0.86 & - & - & 1897.04 & -0.03 & 1146.40 & 750 & $B^{1} C^{6} D^{4}$ & 1896 & 4.57 \\
\hline RC105B & 2296.16 & -0.96 & - & - & 51 & -0.58 & 1530.28 & 744 & $A^{1} B^{6} C^{6} D^{2}$ & 2274 & 5.69 \\
\hline RC106B & 57.84 & -1.21 & - & - & 36 & -0 & 1400.13 & 732 & $A^{1} B^{2} C^{8} D^{2}$ & 2132 & 3.12 \\
\hline RC107B & 2008.02 & -1.18 & - & - & 34 & - & 1252.67 & 732 & $A^{1} B^{2} C^{5} D^{1}$ & 198 & 2.45 \\
\hline RC108B & 1920.91 & -1.32 & - & - & 6 & -0.16 & 1133.97 & 762 & $B^{1} C^{6} D^{4}$ & 189 & 2.67 \\
\hline R201B & 1687.44 & -2.47 & - & - & .78 & 0.00 & 1196.78 & 450 & $A^{5}$ & 16 & 6.79 \\
\hline R202B & 1527.74 & -1.73 & - & - & 1508.16 & -0.42 & 1051.81 & 450 & $A^{5}$ & 150 & 7.23 \\
\hline R203B & 1379.15 & -2.84 & - & - & 1341.09 & 0.00 & 891.092 & 450 & $A^{5}$ & 134 & 4.56 \\
\hline R204B & 1243.56 & -2.09 & - & - & 1218.14 & 0.00 & 768.14 & 450 & $A^{5}$ & 121 & 4.11 \\
\hline R205B & 1471.97 & -3.60 & - & - & 1418.97 & 0.13 & 970.81 & 450 & $A^{5}$ & 142 & 6.47 \\
\hline R206B & 00.84 & -3.97 & - & - & 16.34 & 0.08 & 897.41 & 450 & $A^{5}$ & 134 & 6.99 \\
\hline R207B & 1333.53 & -4.30 & - & - & 1277.58 & 0.08 & 828.57 & 450 & $A^{5}$ & 127 & 6.78 \\
\hline R208B & 1225.37 & -2.23 & - & - & 1197.24 & 0.12 & 748.6 & 450 & $A^{5}$ & 1198.70 & 5.47 \\
\hline R209B & 1370.30 & -3.62 & - & - & 1322.42 & 0.00 & 872.42 & 450 & $A^{5}$ & 1322.42 & 5.47 \\
\hline $\mathrm{R} 210 \mathrm{~B}$ & 1418.54 & -3.51 & - & - & 1374.31 & -0.28 & 920.41 & 450 & $A^{5}$ & $1370.41^{*}$ & 5.93 \\
\hline R211B & 1263.72 & -3.54 & - & - & 1219.93 & 0.05 & 770.57 & 450 & $A^{5}$ & 1220.57 & 7.81 \\
\hline C201B & 1700.87 & -0.35 & - & - & 1695.02 & 0.00 & 695.02 & 1000 & $A^{5}$ & 1695.02 & 2.11 \\
\hline C202B & 1687.84 & -0.15 & - & - & 1685.24 & 0.00 & 685.24 & 1000 & $A^{5}$ & 1685.24 & 2.33 \\
\hline C203B & 996.25 & -0.87 & - & - & 1681.55 & 0.00 & 681.55 & 1000 & $A^{5}$ & 1681.55 & 2.57 \\
\hline C204B & 1705.94 & -1.69 & - & - & 1677.66 & 0.00 & 677.66 & 1000 & $A^{5}$ & 1677.66 & 3.69 \\
\hline C205B & 1711.00 & -1.16 & - & - & 1691.36 & 0.00 & 691.36 & 1000 & $A^{5}$ & 1691.36 & 3.07 \\
\hline C206B & 1691.70 & -0.14 & - & - & 1689.32 & 0.00 & 689.32 & 1000 & $A^{5}$ & 1689.32 & 3.19 \\
\hline C207B & 1704.88 & -1.04 & - & - & 1687.35 & 0.00 & 687.35 & 1000 & $A^{5}$ & 1687.35 & 3.76 \\
\hline $\mathrm{C} 208 \mathrm{E}$ & 89.59 & -0.18 & - & - & 1686.50 & 0.00 & 686.50 & 1000 & $A^{5}$ & 1686.50 & 2.41 \\
\hline $\mathrm{RC} 201 \mathrm{~B}$ & 65.31 & -1.24 & - & - & 1938.36 & 0.1 & 1321.16 & 620 & $A^{4} B^{1} C^{4}$ & 1941.16 & 6.98 \\
\hline $\mathrm{RC} 202 \mathrm{~B}$ & 71.87 & -0.22 & - & - & 1772.81 & -0.27 & 1128.04 & 640 & $A^{1} B^{1} C^{5}$ & $1768.04^{*}$ & 6.47 \\
\hline RC203B & 19.55 & -1.00 & - & - & 1604.04 & -0.03 & 943.548 & 660 & $A^{1} B^{1} C^{5}$ & $1603.55^{*}$ & 6.15 \\
\hline RC204B & 01.10 & -0.79 & - & - & 1490.25 & -0.07 & 829.27 & 660 & $C^{6}$ & $1489.27^{*}$ & 3.47 \\
\hline RC205B & 53.58 & -1.10 & - & - & 1832.53 & 0.04 & 1193.34 & 640 & $A^{1} B^{7} C^{1}$ & 1833.34 & 3.98 \\
\hline RC206B & 61.49 & -2.15 & - & - & 1725.44 & -0.06 & 1074.41 & 650 & $A^{3} B^{1} C^{3} D^{1}$ & $1724.41^{*}$ & 4.54 \\
\hline RC207B & 1666.03 & -0.96 & - & - & 1646.37 & 0.23 & 1000.23 & 650 & $B^{3} C^{4}$ & 1650.23 & 5.01 \\
\hline RC208B & 1494.11 & -0.83 & - & - & 1483.20 & -0.1 & 821.743 & 660 & $C^{6}$ & 1481.74* & 4.08 \\
\hline
\end{tabular}


Table A.6: Results for FD for cost structure $C$

\begin{tabular}{|c|c|c|c|c|c|c|c|c|c|c|c|}
\hline \multirow[t]{2}{*}{ Instance set } & \multicolumn{2}{|l|}{ MDA } & \multicolumn{2}{|l|}{ BPDRT } & \multicolumn{2}{|l|}{ UHGS } & \multicolumn{5}{|l|}{ HEA } \\
\hline & $\mathrm{TC}$ & Dev & $\mathrm{TC}$ & Dev & $\mathrm{TC}$ & Dev & $\mathrm{DC}$ & $\mathrm{VC}$ & Mix & $\mathrm{TC}$ & Time \\
\hline R101C & 1951.20 & -0.71 & 1951.89 & -0.75 & 1951.20 & -0.71 & 1629.38 & 308 & $A^{1} B^{8} C^{5} D^{6}$ & 1937.38* & 4.17 \\
\hline R102C & 1770.40 & -0.46 & 1778.29 & -0.91 & 1785.35 & -1.31 & 1465.22 & 297 & $A^{2} C^{11} D^{5}$ & $1762.22^{*}$ & 3.23 \\
\hline R103C & 1558.17 & -0.72 & 1555.26 & -0.54 & 1552.34 & -0.35 & 1224.98 & 322 & $A^{1} C^{6} D^{7} E^{1}$ & $1546.98^{*}$ & 3.69 \\
\hline R104C & 1367.82 & -1.14 & 1372.08 & -1.46 & 1355.15 & -0.21 & 1013.37 & 339 & $A^{1} C^{1} D^{5} E^{4}$ & $1352.37^{*}$ & 5.17 \\
\hline R105C & 1696.67 & -0.91 & 1698.26 & -1.00 & 1694.56 & -0.78 & 1381.44 & 300 & $B^{3} C^{4} D^{9}$ & $1681.44^{*}$ & 4.13 \\
\hline R106C & 1589.25 & -0.23 & 1590.11 & -0.28 & 1583.17 & 0.16 & 1274.65 & 311 & $B^{2} C^{5} D^{7} E^{1}$ & 1585.65 & 3.67 \\
\hline R107C & 1435.21 & -0.76 & 1439.81 & -1.08 & 1428.08 & -0.26 & 1080.37 & 344 & $A^{1} C^{1} D^{7} E^{3}$ & $1424.37^{*}$ & 5.98 \\
\hline R108C & 1334.75 & -1.24 & 1334.68 & -1.23 & 1314.88 & 0.27 & 968.444 & 350 & $A^{1} C^{1} D^{5} E^{4}$ & 1318.44 & 4.78 \\
\hline R109C & 1515.22 & -0.54 & 1514.13 & -0.47 & 1506.59 & 0.03 & 1185.1 & 322 & $B^{1} C^{1} D^{10} E^{1}$ & 1507.10 & 4.11 \\
\hline $\mathrm{R} 110 \mathrm{C}$ & 1457.42 & -0.97 & 1461.85 & -1.28 & 1443.92 & -0.04 & 1101.37 & 342 & $B^{1} C^{1} D^{10} E^{1}$ & $1443.37^{*}$ & 4.78 \\
\hline $\mathrm{R} 111 \mathrm{C}$ & 1439.43 & -1.41 & 1439.14 & -1.39 & 1420.15 & -0.05 & 1089.43 & 330 & $A^{1} B^{1} D^{7} E^{3}$ & $1419.43^{*}$ & 5.14 \\
\hline $\mathrm{R} 112 \mathrm{C}$ & 1358.17 & -2.27 & 1343.26 & -1.15 & 1327.58 & 0.03 & 989.01 & 339 & $C^{1} D^{7} E^{3}$ & 1328.01 & 4.67 \\
\hline $\mathrm{C} 101 \mathrm{C}$ & 1628.94 & 0.00 & 1628.94 & 0.00 & 1628.94 & 0.00 & 828.94 & 800 & $B^{10}$ & 1628.94 & 1.99 \\
\hline $\mathrm{C} 102 \mathrm{C}$ & 1597.66 & 0.00 & 1597.66 & 0.00 & 1597.66 & 0.00 & 847.66 & 750 & $A^{1} B^{9}$ & 1597.66 & 2.14 \\
\hline C103C & 1596.56 & 0.00 & 1596.56 & 0.00 & 1596.56 & 0.00 & 846.56 & 750 & $A^{1} B^{9}$ & 1596.56 & 2.65 \\
\hline C104C & 1594.06 & -0.21 & 1590.86 & -0.01 & 1590.76 & 0.00 & 840.76 & 750 & $A^{1} B^{9}$ & 1590.76 & 2.11 \\
\hline C105C & 1628.94 & 0.00 & 1628.94 & 0.00 & 1628.94 & 0.00 & 828.94 & 800 & $B^{10}$ & 1628.94 & 2.41 \\
\hline C106C & 1628.94 & 0.00 & 1628.94 & 0.00 & 1628.94 & 0.00 & 828.94 & 800 & $B^{10}$ & 1628.94 & 1.74 \\
\hline $\mathrm{C} 107 \mathrm{C}$ & 1628.94 & 0.00 & 1628.94 & 0.00 & 1628.94 & 0.00 & 828.94 & 800 & $B^{10}$ & 1628.94 & 2.03 \\
\hline C108C & 1622.75 & 0.00 & 1622.75 & 0.00 & 1622.75 & 0.00 & 892.75 & 730 & $A^{3} B^{8}$ & 1622.75 & 2.56 \\
\hline $\mathrm{C} 109 \mathrm{C}$ & 1614.99 & 0.00 & 1614.99 & 0.00 & 1615.93 & 0.06 & 864.99 & 750 & $A^{1} B^{9}$ & 161 & 2.97 \\
\hline $\mathrm{RC} 101 \mathrm{C}$ & 2048.44 & -0.72 & 2053.55 & -0.97 & 2043.48 & -0.47 & 1637.89 & 396 & $A^{1} B^{6} C^{8} D^{1}$ & $2033.89^{*}$ & 4.16 \\
\hline $\mathrm{RC} 102 \mathrm{C}$ & 1860.48 & -0.68 & 1872.49 & -1.33 & 1847.92 & 0.00 & 1481.92 & 366 & $C^{5} D^{3}$ & 1847 & 4.03 \\
\hline RC103C & 1660.81 & -0.88 & 1663.08 & -1.02 & 1646.35 & 0.00 & 1271.35 & 375 & $C^{8} D^{3}$ & 16 & 4.17 \\
\hline $\mathrm{RC} 104 \mathrm{C}$ & 1536.24 & -1.14 & 1540.61 & -1.43 & 1522.04 & -0.20 & 1143.96 & 375 & $C^{4} D^{6}$ & $1518.96^{*}$ & 5.14 \\
\hline $\mathrm{RC} 105 \mathrm{C}$ & 1913.09 & -1.49 & 1929.89 & -2.39 & 1913.06 & -1.49 & 1497.92 & 387 & $A^{2} B^{3} C^{8} D^{2}$ & $1884.92^{*}$ & 4.57 \\
\hline $\mathrm{RC} 106 \mathrm{C}$ & 1772.05 & -1.03 & 1776.52 & -1.28 & 1770.95 & -0.97 & 1372.99 & 381 & $A^{1} B^{2} C^{8} D^{2}$ & 1753.99* & 3.44 \\
\hline $\mathrm{RC} 107 \mathrm{C}$ & 1615.74 & -0.91 & 1633.29 & -2.01 & 1607.11 & -0.37 & 1211.12 & 390 & $B^{1} C^{6} D^{4}$ & $1601.12 *$ & 3.47 \\
\hline $\mathrm{RC} 108 \mathrm{C}$ & 1527.35 & -0.72 & 1527.87 & -0.76 & 1523.96 & -0.50 & 1126.36 & 390 & $A^{1} C^{4} D^{6}$ & $1516.36^{*}$ & 3.64 \\
\hline $\mathrm{R} 201 \mathrm{C}$ & 1441.46 & -0.84 & 1466.13 & -2.56 & 1443.41 & -0.97 & 1204.50 & 225 & $A^{5}$ & $1429.50 *$ & 4.54 \\
\hline $\mathrm{R} 202 \mathrm{C}$ & 1298.10 & -1.96 & 1296.78 & -1.86 & 1283.16 & -0.79 & 1048.11 & 225 & $A^{5}$ & $1273.11 *$ & 7.12 \\
\hline R203C & 1145.38 & -2.62 & 1127.28 & -1.00 & 1116.09 & 0.00 & 891.09 & 225 & $A^{5}$ & 1116.09 & 4.58 \\
\hline $\mathrm{R} 204 \mathrm{C}$ & 1019.77 & -2.68 & 1000.89 & -0.78 & 993.14 & 0.00 & 768.14 & 225 & $A^{5}$ & 993.14 & 6.81 \\
\hline $\mathrm{R} 205 \mathrm{C}$ & 1222.03 & -2.19 & 1240.74 & -3.76 & 1193.97 & 0.15 & 970.81 & 225 & $A^{5}$ & 1195.81 & 6.21 \\
\hline $\mathrm{R} 206 \mathrm{C}$ & 1138.26 & -1.51 & 1141.13 & -1.76 & 1121.34 & 0.00 & 896.34 & 225 & $A^{5}$ & 1121.34 & 5.14 \\
\hline $\mathrm{R} 207 \mathrm{C}$ & 1086.42 & -3.21 & 1067.97 & -1.46 & 1052.58 & 0.00 & 827.58 & 225 & $A^{5}$ & 1052.58 & 5.23 \\
\hline R208C & 976.11 & -0.25 & 979.50 & -0.60 & 969.90 & 0.39 & 748.70 & 225 & $A^{5}$ & 973.70 & 5.47 \\
\hline R209C & 1140.96 & -4.20 & 1140.96 & -4.20 & 1097.42 & -0.22 & 869.97 & 225 & $A^{5}$ & $1094.97^{*}$ & 5.64 \\
\hline $\mathrm{R} 210 \mathrm{C}$ & 1161.87 & -1.43 & 1170.29 & -2.17 & 1149.85 & -0.38 & 920.48 & 225 & $A^{5}$ & $1145.48^{*}$ & 6.17 \\
\hline $\mathrm{R} 211 \mathrm{C}$ & 1015.84 & -2.10 & 1008.54 & -1.37 & 994.93 & 0.00 & 769.93 & 225 & $A^{5}$ & 994.93 & 6.17 \\
\hline $\mathrm{C} 201 \mathrm{C}$ & 1194.33 & 0.00 & 1194.33 & 0.00 & 1194.33 & 0.00 & 694.33 & 500 & $A^{5}$ & 1194.33 & 4.50 \\
\hline $\mathrm{C} 202 \mathrm{C}$ & 1189.35 & -0.35 & 1185.24 & 0.00 & 1185.24 & 0.00 & 685.24 & 500 & $A^{5}$ & 1185.24 & 2.36 \\
\hline $\mathrm{C} 203 \mathrm{C}$ & 1176.25 & 0.00 & 1176.25 & 0.00 & 1176.25 & 0.00 & 656.25 & 520 & $A^{1} B^{3}$ & 1176.25 & 3.07 \\
\hline $\mathrm{C} 204 \mathrm{C}$ & 1176.55 & -0.10 & 1176.55 & -0.10 & 1175.37 & 0.00 & 675.37 & 500 & $A^{5}$ & 1175.37 & 3.09 \\
\hline $\mathrm{C} 205 \mathrm{C}$ & 1190.36 & 0.00 & 1190.36 & 0.00 & 1190.36 & 0.00 & 690.36 & 500 & $A^{5}$ & 1190.36 & 4.50 \\
\hline C206C & 1188.62 & 0.00 & 1188.62 & 0.00 & 1188.62 & 0.00 & 668.62 & 520 & $A^{1} B^{3}$ & 1188.62 & 3.99 \\
\hline $\mathrm{C} 207 \mathrm{C}$ & 1184.88 & 0.00 & 1187.71 & -0.24 & 1184.88 & 0.00 & 684.88 & 500 & $A^{5}$ & 1184.88 & 3.17 \\
\hline $\mathrm{C} 208 \mathrm{C}$ & 1187.86 & -0.11 & 1186.50 & 0.00 & 1186.50 & 0.00 & 686.50 & 500 & $A^{5}$ & 1186.50 & 2.87 \\
\hline RC201C & 1632.41 & -0.41 & 1630.53 & -0.30 & 1623.36 & 0.14 & 1285.71 & 340 & $A^{1} B^{7} C^{1}$ & 1625.71 & 6.01 \\
\hline $\mathrm{RC} 202 \mathrm{C}$ & 1459.84 & -1.02 & 1461.44 & -1.13 & 1447.27 & -0.15 & 1095.12 & 350 & $A^{1} B^{3} C^{4}$ & $1445.12^{*}$ & 4.12 \\
\hline RC203C & 1295.07 & -1.69 & 1292.92 & -1.52 & 1274.04 & -0.04 & 943.55 & 330 & $B^{3} C^{4}$ & $1273.55^{*}$ & 3.67 \\
\hline $\mathrm{RC} 204 \mathrm{C}$ & 1171.26 & -1.15 & 1162.91 & -0.43 & 1159.00 & -0.09 & 807.94 & 350 & $C^{2} D^{3}$ & $1157.94^{*}$ & 5.14 \\
\hline $\mathrm{RC} 205 \mathrm{C}$ & 1525.28 & -0.66 & 1632.67 & -7.74 & 1512.53 & 0.19 & 1180.34 & 335 & $A^{1} B^{4} C^{3}$ & 1515.34 & 5.01 \\
\hline $\mathrm{RC} 206 \mathrm{C}$ & 1425.15 & -1.84 & 1420.89 & -1.53 & 1395.18 & 0.30 & 1074.41 & 325 & $A^{1} B^{1} C^{5}$ & 1399.41 & 3.27 \\
\hline $\mathrm{RC} 207 \mathrm{C}$ & 1332.40 & -1.13 & 1328.29 & -0.82 & 1314.44 & 0.23 & 987.50 & 330 & $C^{6}$ & 1317.50 & 5.47 \\
\hline RC208C & 1155.02 & -1.31 & 1152.92 & -1.12 & 1140.10 & 0.00 & 790.09 & 350 & $C^{2} D^{3}$ & 1140.10 & 5.99 \\
\hline
\end{tabular}

